TRANSACTIONS OF THE

AMERICAN MATHEMATICAL SOCIETY

Volume 362, Number 12, December 2010, Pages 6591-6618

S 0002-9947(2010)05085-8

Article electronically published on July 20, 2010

\title{
DYNAMICS AND SELF-SIMILARITY IN MIN-DRIVEN CLUSTERING
}

\author{
GOVIND MENON, BARBARA NIETHAMMER, AND ROBERT L. PEGO
}

\begin{abstract}
We study a mean-field model for a clustering process that may be described informally as follows. At each step a random integer $k$ is chosen with probability $p_{k}$, and the smallest cluster merges with $k$ randomly chosen clusters. We prove that the model determines a continuous dynamical system on the space of probability measures supported in $(0, \infty)$, and we establish necessary and sufficient conditions for the approach to self-similar form. We also characterize eternal solutions for this model via a Lévy-Khintchine formula. The analysis is based on an explicit solution formula discovered by Gallay and Mielke, extended using a careful choice of time scale.
\end{abstract}

\section{INTRODUCTION}

1.1. A mean-field model for clustering. The clustering processes we consider are motivated by a simplified model for domain wall motion in the one-dimensional Allen-Cahn equation $\partial_{t} u=\partial_{x x} u+u-u^{3}$. The domain walls become points on the line, and the domains are the intervals separated by these points. The pattern coarsens by a simple rule: At each step, the smallest domain combines with its two neighbors to form a single domain, and this is repeated indefinitely. Computational simulations of this 'min-driven' domain coarsening process indicate that for a considerable variety of initial distributions, the domain size distribution approaches a self-similar form [4, 12.

A mean-field model of this process was derived by Nagai and Kawasaki [12], and it turns out to be amenable to a rigorous analysis aimed at explaining this behavior [4, 8. We consider an infinite number of domains on the line and study the statistics of domain sizes using a number density function $f(t, x)$. We assume that in any interval $I$ of unit length, the expected number of domains with lengths in the range $(x, x+d x)$ is given by $f(t, x) d x$. The expected value of the total number of domains in $I$ is denoted by $N(t)=\int_{0}^{\infty} f(t, x) d x$. We assume that $N(t)$ is finite and denote the associated probability density by

$$
\rho_{t}(x)=\frac{f(t, x)}{N(t)} .
$$

Received by the editors July 28, 2008 and, in revised form, April 15, 2009.

2010 Mathematics Subject Classification. Primary 82C22.

This material is based upon work supported by the National Science Foundation under grant nos. DMS 06-04420, DMS 06-05006, DMS 07-48482, and by the Center for Nonlinear Analysis under NSF grants DMS 04-05343 and 06-35983.

The second and third authors thank the DFG for partial support through a Mercator professorship for RLP at Humboldt University and through the Research Group Analysis and Stochastics in Complex Physical Systems. 
We let $l(t)$ denote the size of the smallest domain at time $t$, so $f(t, x)=0$ for $x<l(t)$. The expected number of coalescence events per unit time is then

$$
f(t, l) \dot{l} \text {. }
$$

The coalescence events affecting domains of size $x$ in the time interval $(t, t+d t)$ are (a) loss: consecutive domains of size $x, l, y$ (or $y, l, x)$ combine to form a domain of size $x+l+y$; (b) gain: domains of size $y, l, x-y-l$ combine to form a domain of size $x$. Under the mean-field assumption that coalescing domains have sizes chosen randomly and independently from the current overall size distribution, these events have respective relative probability densities

$$
\rho_{t}(x) \rho_{t}(y), \quad \rho_{t}(y) \rho_{t}(x), \quad \rho_{t}(y) \rho_{t}(x-y-l) .
$$

The rate equation for the evolution of $f$ is obtained by summing over all loss and gain terms:

$$
\partial_{t} f(t, x)=f(t, l) i\left(\int_{l}^{x-l} \rho_{t}(y) \rho_{t}(x-y-l) d y-2 \rho_{t}(x) \int_{l}^{\infty} \rho_{t}(y) d y\right), \quad x>l .
$$

Clustering phenomena are seen in fields as varied as population genetics and physical chemistry. Thus, while the coarsening of intervals provides concrete motivation, the notion of a 'cluster' can have widely different interpretations in applications. The model (1.3) above is one of a family of what we call min-driven clustering models that can be analyzed together in one setting as in [8. At each step a random integer $k \geq 1$ is chosen with probability $p_{k}$, and the smallest cluster merges with $k$ randomly chosen clusters. The mean-field assumption is that all these random variables are independent. The only assumptions we impose on the probabilities are that

$$
p_{k} \geq 0, \quad \sum_{k=1}^{\infty} p_{k}=1, \quad \sum_{k=1}^{\infty} k p_{k}<\infty .
$$

The evolution of the number density under this process is described by the following rate equation for cluster size density:

$$
\partial_{t} f(t, x)=f(t, l) i \sum_{k=1}^{\infty} p_{k}\left(\rho_{t}^{\star k}(x-l)-k \rho_{t}(x)\right), \quad x>l(t) .
$$

Here the notation $\rho_{t}^{\star k}$ denotes $k$-fold self-convolution. Equation (1.5) is obtained by summing over all loss and gain events as in (1.3): the $k$-th term in the sum is the probability of the growth and loss of a cluster of size $x$ in a merger of the smallest cluster with $k$ independently chosen clusters. The sum over $k$ with weight $p_{k}$ corresponds to the probability of choosing the independent random integer $k$. One obtains (1.3) if $p_{2}=1$. The case $p_{1}=1$ corresponds to a "paste-all" model discussed by Derrida et al. 6]. Also see [9] for a model of social conflict with rather similar solution formulas.

An important feature of the model (1.5) is its invariance under reparametrization in time. If we change variables via $t=T(\tilde{t}), \tilde{f}(x, \tilde{t})=f(x, t), \tilde{l}(\tilde{t})=l(t)$, then equation (1.5) retains its form since

$$
\partial_{\tilde{t}} \tilde{f}(x, \tilde{t})=\dot{T} \partial_{t} f, \quad \partial_{\tilde{t}} \tilde{l}=\dot{T} \partial_{t} l .
$$


A careful choice of the time scale is key to the analysis. In the first mathematical study of (1.3) 4], the authors imposed the relation $f(t, l) i=1$, meaning the number of coalescence events per unit time is constant [4. In a more recent paper, Gallay and Mielke parametrized time by the minimum size, that is, $l(t)=t[8$. This leads to an elegant solution procedure that was used to prove some basic results on well-posedness and the approach to self-similarity. Gallay and Mielke showed that (1.5) defines a strongly continuous flow in $L^{1}$, that (1.5) admits a one-parameter family of self-similar solutions and that suitable initial densities yield convergence to self-similar form. Precise comparisons between these results and ours are made later in this paper.

In this article, we introduce yet another time scale. We parametrize time inversely to the total number of domains, so that

$$
t=N(t)^{-1} \text {. }
$$

We shall argue that this is a natural choice for a number of reasons. It retains the simplicity of the choice $l(t)=t$ and allows us to obtain: (a) existence and uniqueness for measure-valued solutions; (b) necessary and sufficient conditions for convergence to a self-similar form; (c) a characterization of eternal solutions for the dynamical system defined by (1.3). We comment on these in greater depth below.

Let us first explain one simple motivation for (1.6). We show below that for (1.3), $f(t, l) \dot{l}=-\dot{N} / 2$. Thus, $f(t, l) \dot{l}=N^{2} / 2$ when (1.6) holds, and (1.3) now takes the form

$$
\partial_{t} f(t, x)=\frac{1}{2} \int_{l}^{x-l} f(t, y) f(t, x-y-l) d y-f(t, x) \int_{l}^{\infty} f(t, y) d y, \quad x>l .
$$

If we take $l(t) \equiv 0$ (as a model when the smallest domains have negligible size, for example), this reduces to a basic solvable model of clustering: Smoluchowski's coagulation equation with constant kernel. In recent work, we provided a comprehensive analysis of dynamic scaling in this equation by exploiting an analogy with the classical limit theorems of probability theory [10, 11]. We use these insights to guide our study of (1.5).

1.2. Measure-valued solutions. Mean-field models of domain coarsening, such as the LSW model, Smoluchowski's coagulation equation, and (1.5), correspond to physical processes where mass is transported from small to large scales. For several reasons, it is natural to consider measure-valued solutions, for which the size distribution need not have a continuous or integrable density. Such solutions are physically meaningful, as many clustering processes (e.g., polymerization) involve a discrete set of sizes based on an elementary unit. A formulation via measures is also mathematically elegant, as it allows us to unify the treatment of discrete and continuous coagulation models, exploit simple criteria for compactness and continuity, and prove basic uniform estimates.

To any solution of (1.5) we associate a probability measure $F_{t}$ with distribution function written as

$$
F_{t}(x)=\frac{1}{N(t)} \int_{0}^{x} f(t, y) d y=\int_{0}^{x} \rho_{t}(y) d y .
$$

Here we adopt the convention (common in probability theory) of often using the same letter to denote a measure and its distribution function. For any probability distribution $F$ on $[0, \infty)$, we call $l=\inf \{x \mid F(x)>0\}$ the min of $F$. (This is 
short for "minimum size," regarding $F$ as a probability distribution for size.) We will prove that the initial-value problem for an appropriate weak form of (1.5) is well-posed for initial probability measures $F_{t_{0}}$ with positive min. That is, (1.5) with (1.6) determines a continuous dynamical system on the space of probability measures with positive min, equipped with the weak topology. See Theorem 4.4 below. By comparison, Gallay and Mielke established that the initial-value for (1.5) defines a continuous dynamical system on the space of probability densities in $L^{1}(1, \infty)$ equipped with the strong topology [8, theorem. 3.3]. The solutions we construct arise by a natural completion of these $L^{1}$ dynamics.

1.3. Dynamic scaling. A common theme in recent studies of dynamic scaling in mean-field models of coarsening is that the approach to self-similarity is both degenerate and delicate. The problem is degenerate because there is a one-parameter family of self-similar solutions. For the model studied here with $p_{k}=0$ for all $k$ large enough, Gallay and Mielke found a family of self-similar solutions that may be rewritten in the time scale (1.6) in the form

$$
F_{t}(x)=F^{(\theta)}\left(\frac{x}{l^{(\theta)}(t)}\right), \quad l^{(\theta)}(t)=t^{1 / \theta}, \quad \theta \in(0,1], \quad t>0 .
$$

Here $F^{(\theta)}$ is a probability distribution with density $\rho^{(\theta)}$ supported on $[1, \infty)$. The density $\rho^{(\theta)}$ is known explicitly only through its Laplace transform. Only $\rho^{(1)}$ has finite mass (first moment); $\rho^{(1)}(x)$ decays exponentially as $x \rightarrow \infty$. The distributions $F^{(\theta)}$ for $0<\theta<1$ have heavy tails, with $\rho^{(\theta)}(x) \sim c_{\theta} x^{-(1+\theta)}$ as $x \rightarrow \infty$ (see Theorem 5.1).

The problem is delicate because the domains of attraction of the self-similar solutions are determined by the tails of the initial size distribution, in the precise manner explained below. (See [10, 13, for analogous results on the LSW model of Ostwald ripening and Smoluchowski's coagulation equations with solvable kernels.) Gallay and Mielke showed that all densities with finite mass are attracted to the self-similar solution with $\theta=1$. Moreover, for $0<\theta \leq 1$, they showed that if the initial data $\rho_{t_{0}}$ is sufficiently close to $\rho^{(\theta)}$ in a suitable weighted norm, then the rescaled probability density $t \rho_{t}(t x)$ approaches $\rho^{(\theta)}$ with a rate of convergence determined by the weighted norm (see $[8$, theorems $5.5,5.7]$ ). These results provide sufficient conditions for the approach to self-similarity.

Our aim is to establish conditions that are both necessary and sufficient to answer a more general question about arbitrary scaling limits. We characterize the set of all nondegenerate limits under a general rescaling of the form $F_{t}(\lambda(t) x)$, where $\lambda(t)$ is a measurable, positive function such that $\lim _{t \rightarrow \infty} \lambda(t)=\infty$. A limit is non-degenerate if it is suitable data for the initial-value problem. That is, nondegenerate limits are probability distributions with a positive min.

Theorem 1.1. Let $t_{0}>0$, let $F_{t_{0}}$ be an arbitrary probability measure on $(0, \infty)$ with positive min, and let $F_{t}\left(t \geq t_{0}\right)$ be the associated measure-valued solution of (1.5) (see Theorem 4.4).

(i) Suppose there is a measurable, positive function $\lambda(t) \rightarrow \infty$ as $t \rightarrow \infty$ and a probability measure $F_{*}$ with positive min, such that

$$
\lim _{t \rightarrow \infty} F_{t}(\lambda(t) x)=F_{*}(x)
$$


at all points of continuity of $F_{*}$. Then there exists $\theta \in(0,1]$ and a function $L$ slowly varying at infinity such that the initial data $F_{t_{0}}$ satisfies

$$
\int_{0}^{x} y F_{t_{0}}(d y) \sim x^{1-\theta} L(x), \quad \text { as } \quad x \rightarrow \infty .
$$

Moreover, the min $l(t)$ of $F_{t}$ and the rescaling $\lambda(t)$ satisfy

$$
\lambda(t) l_{*} \sim l(t) \sim t^{1 / \theta} \tilde{L}(t), \quad \text { as } t \rightarrow \infty,
$$

where $l_{*}>0$ is the min of $F_{*}$ and $\tilde{L}$ is slowly varying at infinity, related to $L$ by (6.9).

(ii) Conversely, assume that there exists $\theta \in(0,1]$ and a function $L$ slowly varying at infinity such that the initial data satisfies (1.11). Then $l(t)$ satisfies (1.12), and

$$
\lim _{t \rightarrow \infty} F_{t}(l(t) x)=F^{(\theta)}(x), \quad x \in(0, \infty) .
$$

A positive function $L$ is slowly varying at infinity if it is asymptotically flat under rescaling in the sense that $\lim _{x \rightarrow \infty} L(x y) / L(x)=1$ for every $y>0$. For example, all powers and iterates of the logarithm are slowly varying at infinity. These are the class of admissible corrections to the power law $x^{1-\theta}$.

Part (i) of the theorem is an assertion of rigidity of scaling limits. We assume only that $\lambda(t)$ is measurable, positive and $\lim _{t \rightarrow \infty} \lambda(t)=\infty$. It then follows that the limits must define self-similar solutions, and $\lambda(t)$ must be the time scale associated to the self-similar solution, up to a slowly-varying correction. Part (ii) of the theorem and the sufficient conditions of Gallay and Mielke show that the domains of attraction are determined by the tails of the initial data. As a consequence of part (i) of the theorem, the condition (1.11) is optimal.

Gallay and Mielke 8 used a rather delicate Fourier analysis to establish the existence for self-similar solutions by studying their densities. We will use the proof of part (ii) of the theorem above to simplify much of this analysis and extend it to the case when $p_{k} \neq 0$ for infinitely many $k$. In this we are motivated by a certain resemblance of the min-driven model to hydrodynamic limits of what are called $\Lambda$-coalescents in probability theory [2, which are clustering processes involving arbitrarily many multiple collisions. We find a curious fact; namely, when $\sum p_{k} k \log k=\infty$ there is no self-similar solution with finite mass (first moment). Still, the theorem above correctly describes the domains of attraction. Solutions with finite mass approach the self-similar solution with $\theta=1$, but this self-similar solution has infinite mass.

1.4. Eternal solutions. Theorem 1.1 is a particular example of the principle that the asymptotic behavior under rescaling is determined by the tail of the initial distribution. The dynamics exhibit sensitive dependence on initial conditions, as arbitrarily small changes in the tail of the initial data can lead to widely divergent asymptotic behavior. This indicates a kind of chaos, and it is of interest to find a precise formulation. A comprehensive analysis of such phenomena for the solvable cases of Smoluchowski's coagulation equation appears in [11. This analysis is guided by an analogy with the probabilistic notion of infinite divisibility. Let us first describe these results informally.

Clustering is an irreversible process, and in general we do not expect to be able to solve (1.3) backwards in time (for $t<t_{0}$ ). However, the self-similar solutions 
have the remarkable feature that they are defined for all $t>0$. That is, they are divisible under the coalescence process. We call a solution eternal if it is defined on the maximal interval $(0, \infty)$ consistent with (1.6). In probability theory, the infinitely divisible distributions are characterized by the celebrated Lévy-Khintchine formula. In [11] we extended a result of Bertoin [1] showing that the class of eternal solutions to Smoluchowski's coagulation equations is also characterized by a LévyKhintchine formula. Heuristically, this formula describes the emergence of eternal solutions from infinitesimally small clusters at $t=0$. We also showed that the set of all subsequential limits - the scaling attractor - is in a one-to-one correspondence with the eternal solutions. A rigorous description of chaos is based on the fact that nonlinear dynamics on the scaling attractor is reduced to linear scaling dynamics using the Lévy-Khintchine formula.

In this article, we take the first step towards establishing a similar picture for min-driven clustering. Namely, we prove a Lévy-Khintchine formula characterizing all eternal solutions for min-driven clustering (Theorem 7.4). The choice of time scale (1.6) is very convenient for this analysis.

1.5. Outline. The rest of the article is organized as follows. We describe the solution procedure of Gallay and Mielke in the next section and discuss how the number-driven time scale is motivated by the important example of initial data that are monodisperse (a Dirac delta). The treatment here is formal. We establish some analytic prerequisites in Section 3 . This is followed by rigorous results: the proof of well-posedness for measure-valued solutions (see Theorem 4.4) in Section4, the study of self-similar profiles in Section 5, the characterization of domains of attraction in Section 6, and the characterization of eternal solutions in Section 7 .

\section{THE SOLUTION FORMULA FOR MIN-DRIVEN CLUSTERING}

2.1. The generating function and moment identities. As in the theory of branching processes, it is convenient to keep track of the clustering process with a generating function

$$
Q(z)=\sum_{k=1}^{\infty} p_{k} z^{k}
$$

For example, binary clustering as in the Allen-Cahn model corresponds to $Q(z)=$ $z^{2}$. The generating function $Q$ is analytic in the unit disk $\{|z|<1\}$, and absolutely monotone (that is, $Q$ and all its derivatives are positive on $[0,1)$ ). We assume that the expected number of clusters in the mergers, denoted $Q_{1}$, is finite. That is,

$$
Q_{1}=Q^{\prime}(1)=\sum_{k=1}^{\infty} k p_{k}<\infty
$$

We now consider the evolution equation (1.5) with an arbitrary time scale. We define a convolution operator $\mathbb{Q}(\rho)=\sum_{k=1}^{\infty} p_{k} \rho_{t}^{\star k}$ associated to $Q$, and rewrite (1.5) in the form

$$
\partial_{t} f(t, x)=f(t, l) \dot{i}\left(\mathbb{Q}\left(\rho_{t}\right)(x-l)-Q_{1} \rho_{t}(x)\right), \quad x>l(t) .
$$

We extend the evolution equation (2.3) from densities to measures as follows. We consider a number measure $\nu_{t}$ and a probability measure $F_{t}$ that are related to 
the densities (when they exist) by

$$
\nu_{t}(d x)=f(t, x) d x, \quad F_{t}(d x)=\frac{\nu_{t}(d x)}{N(t)}=\rho_{t}(x) d x .
$$

Let $\mathbb{R}_{+}$denote the interval $[0, \infty)$. If $a: \mathbb{R}_{+} \rightarrow \mathbb{C}$ is continuous with compact support, then formally

$$
\frac{d}{d t} \int_{\mathbb{R}_{+}} a(x) f(t, x) d x=\int_{l}^{\infty} a(x) \partial_{t} f(t, x) d x-a(l) f(t, l) i .
$$

We substitute for $\partial_{t} f(t, x)$ using (2.3), (2.4) to obtain the moment identity

$$
\begin{aligned}
& \frac{d}{d t} \int_{\mathbb{R}_{+}} a(x) \nu_{t}(d x) \\
& \quad=f(t, l) i \sum_{k \geq 1} p_{k} \int_{\mathbb{R}_{+}^{k}}\left[a\left(l+\sum_{i=1}^{k} y_{i}\right)-a(l)-\sum_{i=1}^{k} a\left(y_{i}\right)\right] \prod_{i=1}^{k} F_{t}\left(d y_{i}\right) .
\end{aligned}
$$

Some basic properties of the model are obtained by choosing suitable test functions $a$ in (2.5). We set $a(x)=x$ to see that mass is conserved:

$$
\frac{d}{d t} \int_{0}^{\infty} x \nu_{t}(d x)=0
$$

When $a=1$, we obtain the rate of change of the total number of clusters,

$$
\dot{N}=-Q_{1} f(t, l) \dot{l}=-N Q_{1} \rho_{t}(l) \dot{l} \text {. }
$$

We substitute (2.7) in (2.3) to see that $\rho_{t}$ satisfies

$$
\partial_{t} \rho_{t}=\rho_{t}(l) \dot{l} \mathbb{Q}\left(\rho_{t}\right)(x-l), \quad x>l .
$$

Similarly, we use (2.2), (2.5) and (2.7) to obtain the moment identity

$$
\frac{d}{d t} \int_{\mathbb{R}_{+}} a(x) F_{t}(d x)=\rho_{t}(l) i \sum_{k=1}^{\infty} p_{k} \int_{\mathbb{R}_{+}^{k}}\left[a\left(l+\sum_{i=1}^{k} y_{i}\right)-a(l)\right] \prod_{i=1}^{k} F_{t}\left(d y_{i}\right) .
$$

The time scale so far has been arbitrary. Later, we use the above identity and a suitable choice of time scale to develop a weak form of (1.5).

2.2. Gallay and Mielke's solution formula. A remarkable feature of these mindriven clustering models is that the evolution equation admits an elegant solution via the Fourier (or Laplace) transform. Our analysis relies heavily on this solution procedure, due to Gallay and Mielke [8]. The main difference with [8] is that we prefer to use the Laplace transform, denoted by

$$
\bar{\rho}_{t}(q)=\int_{\mathbb{R}_{+}} e^{-q x} \rho_{t}(x) d x, \quad q>0 .
$$

We set $a(x)=e^{-q x}$ in (2.9) to obtain the ordinary differential equation

$$
\partial_{t} \bar{\rho}_{t}(q)=-\left(\rho_{t}(l) \dot{l}\right) e^{-q l}\left(1-Q\left(\bar{\rho}_{t}(q)\right)\right) .
$$

In order to integrate this equation, we define an analytic function $\varphi$ via

$$
\varphi^{\prime}(z)=\frac{Q_{1}}{1-Q(z)}, \quad \varphi(0)=0 .
$$


(This definition of $\varphi$ differs by the factor $Q_{1}$ from that used in [8].) $\varphi$ is strictly increasing on $[0,1)$. In the case of binary clustering, $Q(z)=z^{2}$, and the functions $\varphi$ and $\varphi^{-1}$ are

$$
\varphi(z)=\log \left(\frac{1+z}{1-z}\right), \quad \varphi^{-1}(w)=\tanh \frac{w}{2} .
$$

We substitute (2.12) in (2.11) to obtain

$$
\partial_{t} \varphi\left(\bar{\rho}_{t}(q)\right)=-\left(Q_{1} \rho_{t}(l) i\right) e^{-q l}, \quad q>0 .
$$

The choice of time scale has played no role in the analysis thus far. Gallay and Mielke parametrize time by the minimum cluster size, setting $i=1$. For clarity of notation, we denote this choice of time scale by $\tau$, reverting to the letter $t$ when we introduce the number-driven time scale in (1.6).

With $l(\tau)=\tau$, the value of $\rho_{\tau}$ on the free boundary $x=\tau$ plays an important role in the solution. We use this density to define a measure on $(0, \infty)$ that we call the trace measure $A$, with distribution function written as

$$
A(\tau)= \begin{cases}\alpha_{0}+\int_{\tau_{0}}^{\tau} Q_{1} \rho_{s}(s) d s, & \tau \geq \tau_{0} \\ \alpha_{0}, & \tau<\tau_{0}\end{cases}
$$

Here $\tau_{0}$ denotes the initial time, and $\alpha_{0}$ is any convenient constant. Equation (2.14) may now be rewritten as

$$
\partial_{\tau} \varphi\left(\bar{\rho}_{\tau}(q)\right)=-e^{-q \tau} \frac{d A}{d \tau} .
$$

Fix $\tau_{1}>\tau_{0}$. We integrate (2.16) from $\tau_{0}$ to $\tau_{1}$ to obtain

$$
\varphi\left(\bar{\rho}_{\tau_{1}}(q)\right)-\varphi\left(\bar{\rho}_{\tau_{0}}(q)\right)=-\int_{\tau_{0}}^{\tau_{1}} e^{-q s} A(d s) .
$$

Since $\rho_{\tau}$ is supported in $[\tau, \infty)$, we have the estimate

$$
\bar{\rho}_{\tau}(q) \leq e^{-q \tau} \int_{\tau}^{\infty} \rho_{\tau}(x) d x=e^{-q \tau} .
$$

We now let $\tau_{1} \rightarrow \infty$ in (2.17) to find the Laplace transform of $A$ given by

$$
\bar{A}(q)=\int_{\mathbb{R}_{+}} e^{-q s} A(d s)=\varphi\left(\bar{\rho}_{\tau_{0}}(q)\right) .
$$

Thus, the trace measure $A$ is the inverse Laplace transform of $\varphi\left(\bar{\rho}_{\tau_{0}}\right)$ and is determined completely by the initial data.

We may now repeat this argument to determine the solution at any time $\tau>\tau_{0}$. We replace $\tau_{0}$ by $\tau$ in (2.17), let $\tau_{1} \rightarrow \infty$, and obtain

$$
\bar{\rho}_{\tau}(q)=\varphi^{-1}\left(\bar{A}_{\tau}(q)\right),
$$

where

$$
\bar{A}_{\tau}(q)=\int_{\tau}^{\infty} e^{-q s} A(d s) .
$$

We note that $\bar{A}_{\tau}$ is the Laplace transform of the truncated trace measure $A_{\tau}$ satisfying

$$
A_{\tau}(d s)=H(s-\tau) A(d s),
$$


where $H$ is the Heaviside function. With the notation $a \vee b=\max (a, b)$, formally we may write

$$
A_{\tau}(s)=A(s) \vee A(\tau)
$$

for the distribution function, making it constant for $s<\tau$. Therefore, the nonlinear evolution of $\rho_{\tau}$ is determined by the linear evolution of $A_{\tau}$. This global linearization underlies the analysis in 8 .

2.3. The number-driven time scale and an extended solution formula. It is natural to try to use the formula (2.19) as a basis for finding measure-valued solutions when the initial data $\rho_{\tau_{0}}$ is replaced by an arbitrary probability distribution. However, we face two difficulties. The first is that it is not clear that (2.19) necessarily defines a measure $\rho_{\tau}$. That is, it is not clear that the right-hand side of (2.19) is necessarily the Laplace transform of a measure. The second difficulty is that whenever the trace measure $A$ has atoms, any solution defined through (2.19) is discontinuous in time, as is clear from (2.20). We overcome the first difficulty by an approximation argument. This relies on the simple and fundamental fact that a limit of completely monotone functions is completely monotone. We overcome the second difficulty by switching to the number-driven time scale (1.6).

Henceforth, the letter $t$ always denotes the number-driven time scale. The measure-valued solution is denoted by $F_{t}$, its Laplace transform by

$$
\bar{F}_{t}(q)=\int_{0}^{\infty} e^{-q r} F_{t}(d r)
$$

and the minimum cluster size by $l(t)$. We use (1.6) and (2.7) to obtain

$$
\rho_{t}(l) i=\frac{1}{Q_{1} t} .
$$

The length, number measure and its Laplace transform in the time scale $t$ are related to solutions in the time scale $\tau$ by

$$
l(t)=\tau, \quad F_{t}(d x)=\rho_{\tau}(x) d x, \quad \bar{F}_{t}(q)=\bar{\rho}_{\tau}(q) .
$$

We now rewrite the solution formula (2.19) in terms of measures. The relation $l(t)=\tau$, equation (2.7) and the definition of $A$ in (2.15) imply

$$
\frac{d t}{t}=Q_{1} \rho_{\tau}(\tau) d \tau=A(d \tau) .
$$

The differential equation (2.11) now takes the form

$$
\partial_{t} \bar{F}_{t}(q)=-\frac{e^{-q l(t)}}{Q_{1} t}\left(1-Q\left(\bar{F}_{t}(q)\right) .\right.
$$

If $t_{0}$ denotes the initial time, we may integrate equation (2.25) to obtain

$$
\log \left(\frac{t}{t_{0}}\right)=A(l(t))-\alpha_{0} .
$$

The change of variables (2.24) and (2.25) affects the Laplace transform of $A_{\tau}$ as follows:

$$
\int_{\tau}^{\infty} e^{-q r} A(d r)=\int_{t}^{\infty} e^{-q l(s)} \frac{d s}{s} .
$$


These calculations yield the following revised solution procedure: Given an arbitrary initial probability measure $F_{t_{0}}$, the trace measure $A$ is found as in (2.18) by inverting its Laplace transform, given by

$$
\bar{A}(q)=\varphi\left(\bar{F}_{t_{0}}(q)\right) .
$$

Next, we determine $l(t)$ through inverting (2.27). Once $l(t)$ is known, the solution $F_{t}$ is determined by inverting the Laplace transform given as in (2.19) and (2.20) by

$$
\bar{F}_{t}(q)=\varphi^{-1}\left(\int_{t}^{\infty} e^{-q l(s)} \frac{d s}{s}\right), \quad t \geq t_{0} .
$$

The main observation is that working with $l(t)$ instead of $A_{\tau}$ yields an evolution continuous in time. Since $l(t)$ is an increasing function, it has at worst jump discontinuities. But (2.30) shows that discontinuities in $l$ do not affect the continuity in $t$ of $\bar{F}_{t}(q)$, and thus the continuity of $F_{t}$ in the weak topology.

2.4. An example: monodisperse initial data. Let us illustrate the meaning of the extended solution formula in the new time scale with an important example. Set $t_{0}=\tau_{0}=1, Q(z)=z^{2}$ and $F_{1}(x)=\mathbf{1}_{x \geq 1}$. That is, initially all clusters have size 1 . Then $\bar{F}_{1}(q)=e^{-q}$, and

$$
\bar{A}(q)=\varphi\left(\bar{F}_{1}(q)\right)=\log \left(1+e^{-q}\right)-\log \left(1-e^{-q}\right) .
$$

We differentiate with respect to $q$ and simplify to obtain

$$
-\partial_{q} \bar{A}(q)=\frac{2 e^{-q}}{1-e^{-2 q}}=2\left(e^{-q}+e^{-3 q}+e^{-5 q}+\cdots\right) .
$$

Since $e^{-k q}$ is the Laplace transform of $\delta_{k}(d x)$, the trace is

$$
A(x)=\sum_{k \leq x, k \text { odd }} \frac{2}{k} \text {. }
$$

$A$ has jump discontinuities at the odd integers. We shall work with the right continuous inverse, so that the minimum cluster size is

$$
l(t)=k, \quad t \in\left[t_{k-2}, t_{k}\right), \quad t_{k}=e^{2\left(1+\frac{1}{3}+\ldots \frac{1}{k}\right)}, \quad k \text { odd },
$$

with $t_{-1}=t_{0}=1$. The solution formula (2.30) now yields

$$
\varphi\left(\bar{F}_{t}(q)\right)=\left(\log \frac{t_{k}}{t}\right) \frac{e^{-k q}}{2}+\frac{e^{-q(k+2)}}{k+2}+\frac{e^{-q(k+4)}}{k+4}+\cdots, \quad t \in\left[t_{k-2}, t_{k}\right) .
$$

The solution has the following interpretation. For $t \in\left[t_{k-2}, t_{k}\right), F_{t}$ is supported on the odd integers greater than or equal to $k$. The fraction of clusters of size $k$ decays continuously to zero over the time interval $\left[t_{k-2}, t_{k}\right)$. Thus, the numberdriven time scale regularizes jumps in the trace measure by providing a finite time for these jumps to vanish. A moment's reflection suggests that this is what we should expect if we approximate the monodisperse data by a smooth density.

\section{ANALYTIC PRELIMINARIES}

This section is a summary of the main analytic methods we use. We present some facts about distribution functions, Laplace transforms and Tauberian theorems in a form suitable for use in later sections. 
3.1. Distribution functions. We will consider measures on an interval $J \subset \mathbb{R}$. We study a measure through its distribution function, often using the same notation for both. It is therefore convenient to introduce the following conventions for brevity. A distribution function $f: J \rightarrow \mathbb{R}$ is a right-continuous, increasing function. ("Increasing" means that $x_{1} \leq x_{2}$ implies $f\left(x_{1}\right) \leq f\left(x_{2}\right)$.) A distribution function $f$ is identified with a measure via

$$
f\left(\left(x_{1}, x_{2}\right]\right)=f\left(x_{2}\right)-f\left(x_{1}\right) .
$$

We do not assume that the function $f$ is positive, since the trace measure for self-similar solutions is of the form $A(\tau)=\theta \log \tau, \tau>0$. Following probabilistic convention, we say a sequence $f_{n}$ of measures on $J$ converges weakly to $f$ (written $\left.f_{n} \rightarrow f\right)$ if and only if $f_{n}([a, b]) \rightarrow f([a, b])$ as $n \rightarrow \infty$ whenever $a, b \in J$ are not atoms of $f$, meaning $f(\{a\})=f(\{b\})=0$. We have $f_{n} \rightarrow f$ if and only if at every point of continuity of $f(x)$,

$$
f_{n}(x)+c_{n} \rightarrow f(x) \text { as } n \rightarrow \infty,
$$

for some constants $c_{n}$ independent of $x$.

Given a distribution function $f: J \rightarrow \mathbb{R}$, its epigraph is the set

$$
\Gamma(f)=\left\{(x, y) \in \mathbb{R}^{2} \mid f\left(x^{-}\right) \leq y \leq f(x), x \in J\right\} .
$$

There is a unique distribution function $f^{\dagger}$, with epigraph obtained by reflection through $x=y$ : $\Gamma\left(f^{\dagger}\right)=\{(x, y) \mid(y, x) \in \Gamma(f)\}$. We call $f^{\dagger}$ the inverse of $f$. We can write

$$
f^{\dagger}(\tau)=\inf \{t \in J \mid f(t)>\tau\}, \quad \tau<\sup \{f(t) \mid t \in J\} .
$$

The following convergence result is not difficult to prove.

Lemma 3.1. Suppose $f_{n}: J \rightarrow \mathbb{R}$ is a sequence of distribution functions that converges to $f$ at all points of continuity. Then for every point of continuity $x$ of $f^{\dagger}$, $f_{n}^{\dagger}(x)$ is defined for sufficiently large $n$, and $\lim _{n \rightarrow \infty} f_{n}^{\dagger}(x)=f^{\dagger}(x)$.

Lemma 3.2. Suppose $f: J \rightarrow \mathbb{R}$ is a distribution function. There exist monotonically increasing and monotonically decreasing sequences of piecewise constant distribution functions that converge to $f$ at all points of continuity.

Proof. We assume that $J=[0, \infty)$ for clarity, and we only construct an increasing sequence of approximations. The argument is easily generalized. We construct a sequence of increasing functions defined via the values of $f$ on a dyadic decomposition of $J$. Precisely, for every positive integer $n$, let $k_{n}(x)$ denote the integer such that $k_{n} \leq x 2^{n}<k_{n}+1$, and set $g_{n}(x)=f\left(k_{n} 2^{-n}\right)$. The value of $g_{n}(x)$ is defined by the left-endpoint of the dyadic endpoint of length $2^{-n}$ that contains $x$. Since $f$ is increasing, we have

$$
g_{1}(x) \leq g_{2}(x) \leq \ldots \leq g_{n}(x) \leq f(x) .
$$

Let us establish convergence of $g_{n}$. Clearly, $\lim _{n \rightarrow \infty} k_{n} 2^{-n}=x$. If $x$ is a point of continuity of $f$, then $g_{n}(x)=f\left(k_{n} 2^{-n}\right)$, and $g_{n}(x) \rightarrow f(x)$. 
3.2. Min history and trace. Our analysis will focus on two related distribution functions: the minimum cluster-size history $l$ and the trace $A$.

Fix $t_{0}>0$. A min history is a positive distribution function $l$ on $\left[t_{0}, \infty\right)$. In this article we also require a min history to be unbounded: $l(t) \rightarrow \infty$ as $t \rightarrow \infty$. Given any min history $l$, we associate a trace $A$ on $(0, \infty)$ via

$$
A(\tau)=\log l^{\dagger}(\tau), \quad \tau>0 .
$$

Note $A(\tau)=\log t_{0}$ for $0<\tau<l\left(t_{0}\right)$ due to (3.2).

Conversely, we say $A$ is a trace if it is a distribution function on $(0, \infty)$ such that (i) $A(\tau)=\log t_{0}$ on some nonempty, maximal interval $\left(0, \tau_{0}\right)$, and (ii) $A(\tau) \rightarrow \infty$ as $\tau \rightarrow \infty$. Given any trace $A$, we can associate a min history $l$ by

$$
l(t)=\exp A^{\dagger}(t), \quad t \geq t_{0} .
$$

Proposition 3.3 (Change of variables). (a) Assume $l$ is a min history and there exists $c \geq 0$ such that

$$
\int_{t_{0}}^{\infty} e^{-q l(t)} \frac{d t}{t}<\infty, \quad q \in(c, \infty) .
$$

Then the trace A given by (3.3) satisfies

$$
\bar{A}(q)=\int_{0}^{\infty} e^{-q \tau} A(d \tau)=\int_{t_{0}}^{\infty} e^{-q l(t)} \frac{d t}{t}, \quad q \in(c, \infty) .
$$

(b) Assume $A$ is a trace such that $\bar{A}(q)<\infty$ for $q \in(c, \infty)$, and let $l(t)$ be defined by (3.4). Then (3.6) holds.

Proof. 1. We first verify the equality for piecewise constant functions. Suppose $0<t_{0}<t_{1}<t_{2}<\ldots$ and $0<l_{0}<l_{1}<\ldots$ are strictly increasing sequences. Consider the piecewise constant, increasing function

$$
l(t)=\sum_{k=0}^{\infty} l_{k} \mathbf{1}_{\left[t_{k}, t_{k+1}\right)}(t), \quad t \geq t_{0}
$$

An associated trace is given by

$$
A(\tau)=\sum_{k=-1}^{\infty} \log t_{k+1} \mathbf{1}_{\left[l_{k}, l_{k+1}\right)}(\tau), \quad \tau \geq 0 .
$$

Here $l_{-1}=0$. Conversely, if $A$ is defined by (3.8), then $l$ is given by (3.7). We fix $q \in(c, \infty)$, assume (3.5), and use (3.7) and (3.8) to compute the integrals in (3.6). Both equal

$$
e^{-q l_{0}} \log \left(\frac{t_{1}}{t_{0}}\right)+e^{-q l_{1}} \log \left(\frac{t_{2}}{t_{1}}\right)+e^{-q l_{2}} \log \left(\frac{t_{3}}{t_{2}}\right)+\cdots .
$$

2. Suppose $l$ is given, and (3.5) holds. Fix $q>0$. We approximate $l$ by a decreasing sequence of piecewise constant functions $l_{n} \downarrow l$. Then $e^{-q l_{n}} \uparrow e^{-q l}$ since $q>c \geq 0$, and moreover we have $A_{n} \uparrow A$ by Lemma 3.1. By the monotone convergence theorem,

$$
\begin{aligned}
\int_{t_{0}}^{\infty} & e^{-q l(t)} \frac{d t}{t}=\lim _{n \rightarrow \infty} \int_{t_{0}}^{\infty} e^{-q l_{n}(t)} \frac{d t}{t} \\
& =\lim _{n \rightarrow \infty} \int_{0}^{\infty} e^{-q \tau} A_{n}(d \tau)=\int_{0}^{\infty} e^{-q \tau} A(d \tau) .
\end{aligned}
$$


3. To prove (b), assume $A$ is such that $\bar{A}(q)<\infty$ for $q \in(c, \infty)$. Consider a sequence of piecewise constant increasing functions $A_{n} \uparrow A$. Then $l_{n} \downarrow l$ and we may apply the monotone convergence theorem again.

Proposition 3.3 and Lemma 3.1 allow us to reformulate the classical equivalence between weak convergence of measures and pointwise convergence of Laplace transforms. The following theorem is a slight modification of [7, XIII.1.2a].

Theorem 3.4. Suppose $l_{n}$ is a sequence of min histories on $\left[t_{0}, \infty\right)$ with associated traces $A_{n}$ that satisfy

$$
\sup _{n} \bar{A}_{n}(q)<\infty, \quad q \in(c, \infty),
$$

for some $c \geq 0$. Then there is a min history $l$ such that $l_{n}(t) \rightarrow l(t)$ as $n \rightarrow \infty$ at all points of continuity if and only if there is a trace $A$ associated to $l$ such that $\bar{A}_{n}(q) \rightarrow \bar{A}(q)$ as $n \rightarrow \infty$, for all $q \in(c, \infty)$.

Proof. Suppose $l_{n} \rightarrow l$. Lemma 3.1 and definition (3.3) then imply the distribution functions $A_{n} \rightarrow A$. By the classical criterion for weak convergence of measures [7, XIII.1.2a], under the hypothesis (3.9) it follows $\bar{A}_{n}(q) \rightarrow \bar{A}(q)$ for all $q>c$.

Conversely, suppose $\bar{A}_{n}(q) \rightarrow \bar{A}(q)$ for all $q>c$, where $A$ is a trace (in particular $A(\tau) \rightarrow \infty$ as $\tau \rightarrow \infty)$. By the classical criterion, the distribution functions $A_{n} \rightarrow$ $A$, and Lemma 3.1 yields $l_{n} \rightarrow l$, where $l$ is given by (3.4).

3.3. Regular variation. A measurable function $L:(0, \infty) \rightarrow(0, \infty)$ is slowly varying at infinity if

$$
\lim _{\lambda \rightarrow \infty} \frac{L(\lambda x)}{L(\lambda)}=1, \quad \text { for every } x>0
$$

A measurable function $R:(0, \infty) \rightarrow(0, \infty)$ is regularly varying at infinity with index $\theta \in \mathbb{R}$ (written $R \in R V_{\theta}$ ) if

$$
\lim _{\lambda \rightarrow \infty} \frac{R(\lambda x)}{R(\lambda)}=x^{\theta}, \quad \text { for every } x>0 .
$$

In this case, $R(x)=x^{\theta} L(x)$, where $L$ is slowly varying at infinity.

The class of regularly-varying functions is remarkably rigid. For example, there is no need to assume that the limit in (3.11) exists for every $x>0$ or that it is a powerlaw. If $R$ is a positive, measurable function on the half-line and $\lim _{\lambda \rightarrow \infty} R(\lambda x) / R(\lambda)$ exists, is positive and finite for $x$ in a set of positive measure, then $f$ is regularly varying at infinity with some index $\theta \in \mathbb{R}$ and the convergence is locally uniform in $x$. This fundamental rigidity lemma (see [7, VIII.8.1] and [3, 1.4.1]) plays a key role in our analysis.

The class $R V_{\theta}$ is of fundamental utility in Tauberian arguments linking a measure $\nu$ on $[0, \infty)$ and its Laplace transform $\bar{\nu}(q)=\int_{0}^{\infty} e^{-q x} \nu(d x)$; see [7, XIII.5.2]:

Theorem 3.5. If $L$ is slowly varying at infinity and $0 \leq \theta<\infty$, then the following are equivalent:

$$
\nu(x) \sim x^{\theta} L(x), \quad x \rightarrow \infty,
$$

and

$$
\bar{\nu}(q) \sim q^{-\theta} L(1 / q) \Gamma(1+\theta), \quad q \rightarrow 0
$$


Moreover, this equivalence remains true when we interchange the roles of the origin and infinity, namely when $x \rightarrow 0$ and $q \rightarrow \infty$.

A refinement of this result will prove useful for us: de Haan's exponential Tauberian theorem [3, theorem 3.9.3]; see [5].

Theorem 3.6. $\exp \nu(x)$ is regularly varying at infinity with index $\theta$ if and only if $\exp \bar{\nu}(q)$ is regularly varying at zero with index $\theta$. If either holds, then

$$
\nu(1 / q)-\bar{\nu}(q) \rightarrow \gamma \theta, \quad q \rightarrow 0,
$$

where $\gamma=0.577215665 \ldots$ is the Euler-Mascheroni constant.

3.4. Rate of divergence in the solution formula. For several reasons, we need to study carefully the asymptotic behavior of $\varphi(z)$ as $z \rightarrow 1$. For this, it is convenient to define $\kappa(q), q \in(0,1)$, via

$$
-\log \kappa(q)=\varphi(1-q)+\log q=\int_{0}^{1-q}\left(\frac{Q_{1}}{1-Q(z)}-\frac{1}{1-z}\right) d z
$$

(note $-\log \kappa(q) \geq 0$ because $Q$ is convex) and set

$$
\kappa_{0}:=\lim _{q \rightarrow 0} \kappa(q) \in[0,1] .
$$

The limit exists because $\kappa$ is decreasing. With this notation $\kappa_{0}$ corresponds to the number $\kappa$ introduced in [8]. For $Q(z)=z^{2}$ we have $\kappa_{0}=\frac{1}{2}$.

We will show that $\kappa_{0}>0$ if and only if $\sum_{k=1}^{\infty}(k \log k) p_{k}<\infty$. Since in 8 , the function $Q$ is a polynomial this finiteness condition is always satisfied. However, we can characterize self-similar solutions and their domains of attraction also in the case $\kappa_{0}=0$.

Lemma 3.7. The function $\kappa(q)$ as defined in (3.14) satisfies $\kappa(q) \rightarrow 0$ as $q \rightarrow 0$ if and only if $\sum_{k=1}^{\infty} p_{k} k \log k=\infty$.

Proof. We compute

$$
R(z):=Q_{1}-\frac{1-Q(z)}{1-z}=\sum_{k=1}^{\infty} p_{k}\left(k-\frac{1-z^{k}}{1-z}\right)=\sum_{k=1}^{\infty} p_{k} \sum_{j=1}^{k-1}\left(1-z^{j}\right) .
$$

Note $R(1)=0$ and the integrand in (3.14) is

$$
\frac{R(z)}{1-Q(z)}=\frac{R(z)}{1-z} \cdot \frac{1}{Q_{1}-R(z)}=\frac{R(z)}{1-z} \cdot \frac{1}{Q_{1}+o(1)}
$$

as $z \rightarrow 1^{-}$. Thus it suffices to show $\int_{0}^{1} R(z) d z /(1-z)<\infty$ if and only if $\sum p_{k} k \log k<\infty$. We observe

$$
\int_{0}^{1} \sum_{j=1}^{k-1} \frac{1-z^{j}}{1-z} d z=\sum_{j=1}^{k-1} \sum_{l=1}^{j} \frac{1}{l}=\sum_{l=1}^{k-1} \sum_{j=l}^{k-1} \frac{1}{l}=\sum_{l=1}^{k-1} \frac{k-l}{l} \sim k \log k
$$

as $k \rightarrow \infty$, whence the desired result follows from (3.16).

Lemma 3.8. The function $\kappa(q)$ is slowly varying as $q \rightarrow 0$. 
Proof. We need to show that $-\log (\kappa(\lambda q) / \kappa(q)) \rightarrow 0$ as $q \rightarrow 0$ for any $\lambda>0$. From (3.16) and (3.17) above, we see that if we replace the integrand in (3.14) by $R(z) /(1-z)$, then in integrating from $1-\lambda q$ to $1-q$ the error is only $o(1)$ as $q \rightarrow 0$. Hence by (3.16),

$$
-\log \frac{\kappa(\lambda q)}{\kappa(q)}=\sum_{k=1}^{\infty} p_{k} k \sum_{l=1}^{k-1}\left(\frac{(1-\lambda q)^{l}}{l}-\frac{(1-q)^{l}}{l}\right)+o(1)
$$

as $q \rightarrow 0$. We consider without loss of generality that $\lambda>1$ and estimate

$$
\sum_{l=1}^{k-1} \frac{(1-\lambda q)^{l}}{l}-\frac{(1-q)^{l}}{l}=\int_{1-\lambda q}^{1-q} \sum_{l=1}^{k-1} x^{l} d x=\int_{1-\lambda q}^{1-q} \frac{1-x^{k-1}}{1-x} d x \leq \log \lambda .
$$

On the other hand,

$$
\sum_{l=1}^{k-1} \frac{(1-\lambda q)^{l}}{l}-\frac{(1-q)^{l}}{l} \rightarrow 0
$$

as $q \rightarrow 0$ for any $k$, and thus the claim follows from the dominated convergence theorem.

\section{WELL-POSEDNESS FOR MEASURES}

In this section, we first work with the min-driven time scale $l(\tau)=\tau$ used by Gallay and Mielke. We prove that an initial continuous probability density $\hat{\rho}$ with $\min \tau_{0}>0$ defines a solution to (2.8). This is a weaker form of the well-posedness theorem of [8]. It is included for its simplicity, and because it is the basis for weak solutions. We then switch to the number-driven time scale (1.6) and use the moment identity (2.9) to show that (2.8) defines a continuous dynamical system on the space of probability measures $\mathcal{P}\left(\mathbb{R}_{+}\right)$.

4.1. Classical solutions. The solution formulas of section 2.2, while explicit, are not immediately suited for the construction of solutions. The main difficulty is to show that positive initial data yields a positive solution. We construct solutions by rewriting (2.8) in integral form with initial data $\hat{\rho}$ for $\tau=\tau_{0}$ as

$$
\begin{aligned}
& \rho_{\tau}(x)=\hat{\rho}(x)+\int_{\tau_{0}}^{\tau} \rho_{s}(s) \mathbb{Q}\left(\rho_{s}\right)(x-s) d s, \quad x>\tau, \\
& \rho_{\tau}(x)=0, \quad x<\tau .
\end{aligned}
$$

We then have

Theorem 4.1. Suppose $\hat{\rho}$ is a continuous probability density with positive min $\tau_{0}$. There exists a unique solution to (4.1) on $\left[\tau_{0}, \infty\right)$ such that $\rho_{\tau_{0}}=\hat{\rho}$ and the solution has the following properties.

(a) For every $\tau \geq \tau_{0}, \rho_{\tau}$ is a continuous probability density with min $\tau$.

(b) The solution formula (2.19) holds for every $q \geq 0, \tau \geq \tau_{0}$.

Proof. We sketch a proof of existence similar to the direct approach in [4 for $Q(z)=z^{2}$ using a different time scale. We fix $\tau_{0}>0$ and let $\hat{\rho}$ be given. Note that since the solution is to satisfy $\rho_{\tau}(x)=0$ for $x<\tau$, the convolution term on the righthand side of (4.1) will depend only upon values of $\rho_{\tau}(y)$ for $\tau_{0}<y<x-\tau \leq x-\tau_{0}$. In particular, this convolution term vanishes for $x<2 \tau_{0}$. 
This means we can construct the solution for $\tau_{0}<\tau<2 \tau_{0}$ by an inductive procedure as follows: For $\tau_{0}<\tau \leq x<2 \tau_{0}$ we have $\rho_{\tau}(x)=\hat{\rho}(x)$ and in particular $\rho_{\tau}(\tau)=\hat{\rho}(\tau)$. For $\tau_{0}<\tau \leq 2 \tau_{0}$, successively on strips $x \in\left[k \tau_{0},(k+2) \tau_{0}\right)$, for $k=2,4, \ldots$, by simple integration in time we can now compute $\rho_{\tau}(x)$ from (4.1), where the right-hand side is always known from a previous step. This determines $\rho_{\tau}(x)$ for $\tau_{0} \leq \tau \leq 2 \tau_{0}$ and all $x$.

To determine the solution globally for all $\tau>\tau_{0}$, the idea is to replace $2 \tau_{0}$ by $\tau_{0}$ and repeat. But in order to justify this we need to verify that $\rho_{\tau}$ remains integrable and conserves total probability. In particular we need to justify (2.11). Let us introduce the distribution function

$$
R_{\tau}(x)=\int_{0}^{x} \rho_{\tau}(y) d y=\int_{\tau}^{x} \rho_{\tau}(y) d y .
$$

This is the probability that a domain has size $\leq x$ at time $t$. Note that for any two distribution functions $R(x), \hat{R}(x)$ on $[0, \infty)$ we have

$$
R \star \hat{R}(x)=\int_{0}^{x} R(x-y) \hat{R}(d y) \leq \int_{0}^{x} R(x) \hat{R}(d y)=R(x) \hat{R}(x) .
$$

Integrating the convolution term in the integrand of (4.1), we find

$$
\int_{0}^{x} \mathbb{Q}\left(\rho_{\tau}\right)(y-\tau) \mathrm{d} y=\sum_{k=1}^{\infty} p_{k} R_{\tau}^{\star k}(x-\tau) \leq \sum_{k=1}^{\infty} p_{k} R_{\tau}(x)^{k}=Q\left(R_{\tau}(x)\right) .
$$

Then it follows $\partial_{\tau} R_{\tau}(x) \leq \rho_{\tau}(\tau)\left(Q\left(R_{\tau}(x)\right)-1\right)$, and since $\rho_{\tau}(\tau) \geq 0$ and $R_{\tau}(x) \leq 1$ initially, $R_{\tau}(x)$ is decreasing in $\tau$ for fixed $x$. It follows $R_{\tau}(\infty) \leq 1$, and so the Laplace transform

$$
\bar{R}_{\tau}(q)=\int_{0}^{\infty} e^{-q x} R_{\tau}(\mathrm{d} x)=\int_{\tau}^{\infty} e^{-q x} \rho_{\tau}(x) \mathrm{d} x
$$

is well defined and $\leq e^{-q \tau}$. Since $\partial_{\tau} R_{\tau}(x)$ is continuous in $\tau$ for all $x, \bar{R}_{\tau}(q)$ is $C^{1}$ in $\tau$ for all $q>0$. This justifies (2.11) and the computations leading up to (2.18) and the solution formula (2.19). From (2.18) we deduce that since $\bar{R}_{\tau_{0}}(0)=1$, $\bar{A}\left(0^{+}\right)=\infty=\bar{A}_{\tau}\left(0^{+}\right)$and then (2.19) yields $\bar{R}_{\tau}\left(0^{+}\right)=1=R_{\tau}(\infty)$, proving that $\rho_{\tau}$ is a probability density for all $\tau$.

The next lemma provides a uniform estimate for smooth approximations.

Lemma 4.2. Suppose $\hat{\rho}$ is a continuous probability density with positive min $\tau_{0}$ and $\rho_{\tau}$ is the solution to (4.1) with initial data $\hat{\rho}$. Then (with $\alpha_{0}=0$ in (2.15) ),

$$
A(\tau) \leq \frac{Q_{1}}{\log 2} \log \left(\frac{2 \tau}{\tau_{0}}\right)
$$

Consequently,

$$
\frac{l(t)}{l\left(t_{0}\right)} \geq \frac{1}{2}\left(\frac{t}{t_{0}}\right)^{(\log 2) / Q_{1}}, \quad t \geq t_{0} .
$$

Proof. If $m$ is an integer such that $\tau \in\left[2^{m-1} \tau_{0}, 2^{m} \tau_{0}\right)$, we divide the domain of integration $\left[\tau_{0}, \tau\right)$ into $m$ pieces and use the fact that $\rho_{s}(s)=\rho_{r}(s)$ for $\tau_{0} \vee \frac{1}{2} s \leq$ 
$r \leq s$ to obtain

$$
\begin{aligned}
Q_{1}^{-1} A(\tau) & =\int_{\tau_{0}}^{2 \tau_{0}} \rho_{\tau_{0}}(s) d s+\int_{2 \tau_{0}}^{2^{2} \tau_{0}} \rho_{2 \tau_{0}}(s) d s+\cdots+\int_{2^{m-1} \tau_{0}}^{\tau} \rho_{2^{m-1} \tau_{0}}(s) d s \\
& \leq m \leq \frac{\log \left(\tau / \tau_{0}\right)}{\log 2}+1 .
\end{aligned}
$$

4.2. Weak solutions. We now switch to the number-driven time scale $N(t)=1 / t$. In order to define weak solutions, we fix a test function $a$, substitute (2.23) in the moment identity (2.9) and integrate in time between $t_{0}$ and $t$ to obtain

$$
\begin{aligned}
\int_{\mathbb{R}_{+}} & a(x) F_{t}(d x)-\int_{\mathbb{R}_{+}} a(x) F_{t_{0}}(d x) \\
& =\int_{t_{0}}^{t} \sum_{k=1}^{\infty} p_{k} \int_{\mathbb{R}_{+}^{k}}\left[a\left(l(s)+\sum_{i=1}^{k} y_{i}\right)-a(l(s))\right] \prod_{i=1}^{k} F_{s}\left(d y_{i}\right) \frac{d s}{Q_{1} s} .
\end{aligned}
$$

We will consider continuous test functions with $\lim _{x \rightarrow \infty} a(x)=0$. Let $C_{0}\left(\mathbb{R}_{+}\right)$ denote the space of such functions with the topology of uniform convergence. Let $\mathcal{P}\left(\mathbb{R}_{+}\right)$denote the space of probability measures on $\mathbb{R}_{+}$equipped with the weak topology. Assume $t_{0}>0$ is fixed.

Definition 4.3. Let $J \subset(0, \infty)$ be an interval. We say that a map $F: J \rightarrow \mathcal{P}\left(\mathbb{R}_{+}\right)$ is a weak solution for min-driven clustering on $J$ if

(1) The map $t \mapsto \int_{\mathbb{R}_{+}} a(x) F_{t}(d x)$ is measurable for every $a \in C_{0}\left(\mathbb{R}_{+}\right)$.

(2) The min of $F_{t}$, denoted $l(t)$, is positive and increasing.

(3) The moment identity (4.5) holds for each $a \in C_{0}\left(\mathbb{R}_{+}\right)$and $t, t_{0} \in J$.

Theorem 4.4. $\quad$ (a) Suppose $\hat{F} \in \mathcal{P}\left(\mathbb{R}_{+}\right)$has positive min, and $t_{0}>0$. Then there is a weak solution $F$ for min-driven clustering on $\left[t_{0}, \infty\right)$ with $F_{t_{0}}=\hat{F}$. Moreover, the min $l(t)$ of $F_{t}$ satisfies (4.4).

(b) The solution in (a) is unique on $\left[t_{0}, t_{1}\right]$ for any $t_{1}>t_{0}$.

(c) Let $\hat{F}^{(n)}$ be a sequence in $\mathcal{P}\left(\mathbb{R}_{+}\right)$with positive min and $F^{(n)}$ the weak solutions with $F_{t_{0}}^{(n)}=\hat{F}^{(n)}$. Assume $\lim _{n \rightarrow \infty} \hat{F}^{(n)}=\hat{F}$ and the limit has positive min. Then $F_{t}^{(n)} \rightarrow F_{t}$ for every $t>t_{0}$.

Proof. 1. It follows from Weierstrass' approximation theorem that finite linear combinations $\sum_{k=1}^{N} c_{k} e^{-k x}$ are dense in $C_{0}\left(\mathbb{R}_{+}\right)$. Therefore, in order to verify the moment identity, it is sufficient to consider the test functions $e^{-q x}, q>0$. Thus, to prove existence of a weak solution on $\left[t_{0}, \infty\right)$, it suffices to construct $F$ weakly continuous such that the Laplace transform satisfies the solution formula (2.30).

2. Let $\tau_{0}$ denote the min of $\hat{F}$. We approximate $\hat{F}$ by a sequence of continuous probability densities $\hat{\rho}^{(n)}$ with $\min \tau_{0}^{(n)}$ with $\lim _{n \rightarrow \infty} \tau_{0}^{(n)}=\tau_{0}$. We further assume that $\hat{\rho}^{(n)}$ is strictly positive on $\left[\tau_{0}^{(n)}, \infty\right)$. It is immediate from (4.1) that the solutions $\rho_{\tau}^{(n)}$ are strictly positive on $[\tau, \infty)$. The trace for these solutions, $A^{(n)}$ is obtained from (2.15) with $\tau_{0}$ replaced by $\tau_{0}^{(n)}, \rho$ by $\rho^{(n)}$ and the choice $\alpha_{0}=\log t_{0}$. $A^{(n)}$ is continuous and strictly increasing, thus so are the min histories $l^{(n)}$. We change variables from the solution formula (2.19) to (2.30) to obtain

$$
\bar{\rho}_{\tau}^{(n)}(q)=\bar{F}_{t}^{(n)}(q)=\varphi^{-1}\left(\int_{t}^{\infty} e^{-q l^{(n)}(s)} \frac{d s}{s}\right), \quad t \geq t_{0} .
$$


3. As $n \rightarrow \infty$, we have $\overline{\hat{\rho}}^{(n)}(q) \rightarrow \overline{\hat{F}}(q)$ for every $q>0$ and $0<\overline{\hat{F}}(q)<1$ for $q>$ 0 . The measures $A^{(n)}$ are supported on $\left[\tau_{0}^{(n)}, \infty\right)$ and satisfy $\bar{A}^{(n)}(q)=\varphi\left(\bar{F}_{t_{0}}^{(n)}(q)\right)$. Therefore,

$$
\lim _{n \rightarrow \infty} \bar{A}^{(n)}(q)=\varphi(\overline{\hat{F}}(q)), \quad q>0 .
$$

It follows that the traces $A^{(n)}$ converge weakly to a trace $A$ supported on $\left[\tau_{0}, \infty\right)$ that satisfies $\bar{A}(q)=\varphi(\overline{\hat{F}}(q))$. Therefore, by Theorem 3.4 the min histories $l^{(n)}$ converge to $l$, the min history associated to $A$. Since $l^{(n)}$ satisfy the uniform estimate (4.4), we may use the dominated convergence theorem to assert

$$
\lim _{n \rightarrow \infty} \bar{F}_{t}^{(n)}(q)=\varphi^{-1}\left(\int_{t}^{\infty} e^{-q l(s)} \frac{d s}{s}\right), \quad t \geq t_{0} .
$$

This shows that for every $t \geq t_{0}$ the measures $F_{t}^{(n)}$ converge weakly to a measure $F_{t}$ that satisfies (2.30). Then $F_{t}$ is a probability measure since $\bar{F}_{t}(0+)=\varphi^{-1}(\infty)=1$. It similarly follows from (2.30) that $F_{t} \rightarrow F_{t_{1}}$ as $t \rightarrow t_{1}$ for every $t_{1} \in\left[t_{0}, \infty\right)$. This completes the proof of part (a), except that it remains to show that $l(t)$ is in fact the min of $F_{t}$.

4. For (b) it suffices to prove uniqueness on $\left[t_{0}, t_{1}\right]$ for some $t_{1}>t_{0}$. The key is to prove uniqueness of the min $l(t)$ on such a time interval, since then uniqueness of $F_{t}$ is easy to establish via the Laplace transform. Note that any weak solution is weakly continuous in time, since the right-hand side of (4.5) is Lipschitz continuous for any test function $a \in C^{0}\left(\mathbb{R}_{+}\right)$. Then since $F_{t_{0}}(x)>0$ for all $x>\tau_{0}$, the min $l(t)$ is right continuous at $t_{0}$, so there exists $t_{1}>t_{0}$ with $\tau_{0} \leq \tau_{1}=l\left(t_{1}\right)<2 \tau_{0}$. Now the idea is that for clusters of size less than $2 \tau_{0}$, there is no gain, only loss. We claim that

$$
F_{t}(x)=\left(F_{t_{0}}(x)-\frac{1}{Q_{1}} \log \left(\frac{t}{t_{0}}\right)\right)_{+}, \quad t \in\left[t_{0}, t_{1}\right], \quad x \in\left[\tau_{0}, 2 \tau_{0}\right) .
$$

This follows by first considering points $x$ of continuity of both $F_{t}$ and $F_{t_{0}}$ such that $l(t)<x<2 \tau_{0}$, so $F_{t}(x)>0$; approximate $\mathbf{1}_{[0, x)}$ by continuous test functions $a$ supported in $\left[0,2 \tau_{0}\right)$. By right continuity and the definition of min, (4.7) follows for all $x \in\left[l(t), 2 \tau_{0}\right)$, and $F_{t}(x)=0$ for $x<l(t)$. Now by weak continuity in time, we infer that with

$$
\hat{t}(x)=t_{0} \exp \left(Q_{1} \hat{F}(x)\right),
$$

for each point of continuity of $\hat{F}$ in $\left(\tau_{0}, \tau_{1}\right]$ we have $l(t)<x$ for $t<\hat{t}(x)$, and $x<l(t)$ for $\hat{t}(x)<t$. Now clearly $l(t)$ is uniquely determined by $\hat{F}$, since it is locally the inverse of $\hat{t}$. It follows from taking the Laplace transform that the min history $l(t)$ constructed in (a), that satisfies (2.30), agrees with the min of $F_{t}$.

5. Part (c) is proven by an argument very similar to Step 2 above.

Scaling. We note for use below the following scaling property that follows easily from the moment identity (4.5). Let $a, b>0$. If $F$ is a weak solution for min-driven clustering on an interval $J$, then $\hat{F}$ is a weak solution on $J / a$, where

$$
\hat{F}_{t}(x)=F_{a t}(b x), \quad t \in J / a, x \geq 0 .
$$




\section{Self-Similar SOlutions}

Let us recall that Gallay and Mielke have classified the self-similar solutions as in (1.9) in the case when $p_{k}=0$ for all large $k$. Here we will recover and extend the basic existence results and classify all domains of attraction by simple means based on the Laplace transform and Tauberian arguments.

Existence. In terms of the distribution function $F_{t}$, self-similar solutions are of the form $F_{t}(x)=F_{*}(x / l(t))$ for some distribution function $F_{*}$ with positive min $(=1)$ and some min history $l(t)$. Without loss of generality we can assume $t_{0}=1$ and $l\left(t_{0}\right)=1$. From (2.30) we obtain for the Laplace transform of $F_{*}$ that

$$
\bar{F}_{*}(q)=\bar{F}_{t}\left(\frac{q}{l(t)}\right)=\varphi^{-1}\left(\int_{t}^{\infty} e^{-q \frac{l(s)}{l(t)}} \frac{d s}{s}\right)=\varphi^{-1}\left(\int_{1}^{\infty} e^{-q \frac{l(t s)}{l(t)}} \frac{d s}{s}\right) .
$$

Hence we conclude that the min history of self-similar solutions must satisfy $l(t s) / l(t)=g(s)$ for some function $g(s)$. Since $l$ is also positive, increasing and nonconstant, we conclude (see [7, VIII.8.1], or subsection 6.1 below) that necessarily, for some $\theta>0, l(s)=s^{1 / \theta}$ and that $F_{*}$ must be a distribution $F^{(\theta)}$ that satisfies

$$
\bar{F}^{(\theta)}(q)=\varphi^{-1}(\theta \operatorname{Ei}(q)),
$$

where $\operatorname{Ei}(q)=\int_{q}^{\infty} e^{-s} d s / s$ denotes the exponential integral. Provided such a distribution $F^{(\theta)}$ does exist, then $\bar{F}_{t}(q)=\bar{F}^{(\theta)}\left(t^{1 / \theta} q\right)$ satisfies (2.30) and is continuous in time, hence determines a self-similar solution by step 1 of the proof of Theorem 4.4. The corresponding trace measures are given by

$$
A^{(\theta)}(\tau)=\theta \log \tau, \quad \tau \in(0, \infty) .
$$

As a by-product of the characterization of scaling limits in section 6, we will prove the existence of distributions $F^{(\theta)}$ that satisfy (5.2) for $0<\theta \leq 1$, and that $\theta \leq 1$ is necessary.

Densities. Next we show that for the self-similar solutions $F^{(\theta)}\left(x / t^{1 / \theta}\right)(0<$ $\theta \leq 1)$, the probability distributions $F^{(\theta)}$ have piecewise smooth densities $\rho^{(\theta)}$ that satisfy an integrodifferential equation,

$$
-\partial_{y}\left(y \rho^{(\theta)}(y)\right)=\frac{\theta}{Q_{1}} \mathbb{Q}\left(\rho^{(\theta)}\right)(y-1), \quad y>1, \quad \rho^{(\theta)}(1)=\frac{\theta}{Q_{1}},
$$

and we study the decay of $\rho^{(\theta)}(y)$ as $y \rightarrow \infty$. When $p_{k}=0$ for all large $k$ (so $Q(z)$ is a polynomial) this has been done by Gallay and Mielke [8. We will recover most of their results by simpler means (the exponential rate of decay for $\theta=1$ is an exception) and extend them to the case when $p_{k}$ is nonzero for infinitely many $k$. It turns out, however, that when $\sum p_{k} k \log k=\infty$, none of the profiles have finite first moment, including the case $\theta=1$.

Let $\theta \in(0,1]$. We claim that the probability distribution $F=F^{(\theta)}$ satisfies the following weak-form profile equation:

$$
\int_{\mathbb{R}_{+}} x a^{\prime}(x) F(d x)=\frac{\theta}{Q_{1}} \sum_{k=1}^{\infty} p_{k} \int_{\mathbb{R}_{+}^{k}}\left(a\left(1+\sum_{j=1}^{k} y_{j}\right)-a(1)\right) \prod_{j=1}^{k} F\left(d y_{j}\right)
$$

for all $C^{1}$ functions $a \in C_{0}\left(\mathbb{R}_{+}\right)$. This follows from the fact that we know $F\left(x / t^{1 / \theta}\right)$ is a weak solution for the min-driven clustering equation (4.5) with $l(t)=t^{1 / \theta}$. 
Changing variables in (4.5) via $x=l(t) \hat{x}$ and similarly for $y_{j}$, we differentiate at $t=1$ to obtain (5.5).

Now, the min of $F$ is 1 (this will be shown in section 6.2 ), so $F$ has density $\rho(x)=0$ on $(0,1)$. Taking $a$ to be supported in $(1,2)$ we find that the righthand side of (5.5) vanishes. Hence restricted to $(1,2)$, the measure $x F(d x)=\beta d x$ for some constant $\beta$, so $F$ has density $\rho(x)=\beta / x$ on $(1,2)$. Taking $a(x)=0$ for $x \leq 1, a(x)=1$ for $x \geq 2$ (approximated by limits; note $\int_{n}^{n+1} x F(d x) \rightarrow 0$ along a subsequence), we find $\beta=\theta / Q_{1}$. Taking $a$ supported in $(0,2)$, we find the right-hand side is $-\beta a(1)$ and we can conclude that 1 is not an atom of $F$.

In this way, proceeding inductively on intervals $(0, n)$ we deduce that $F$ has density $\rho(x)$ satisfying (5.4). When $a$ has support in $(1, n+1)$ the right-hand side depends on the restriction of $F$ to $(0, n)$ where it has density $\rho(x)$, and thus $x F(d x)$ has density $x \rho(x)$ determined by (5.4) on $(1, n+1)$.

Decay. Next we wish to characterize the decay behavior of the densities $\rho^{(\theta)}$. For this we use the properties of the function $\kappa(q)$ which was introduced in (3.14). We denote by $\kappa^{\#}$ the de Bruijn conjugate of $\kappa$. See [3, Sec. 1.5.7]. This is a slowly varying function satisfying

$$
\kappa(q) \kappa^{\#}(q \kappa(q)) \sim 1 \quad \text { as } q \rightarrow 0 .
$$

If $\kappa_{0}>0$, then $\kappa^{\#}\left(0^{+}\right)=\kappa_{0}^{-1}$, and if $\kappa_{0}=0$, then $\kappa^{\#}\left(0^{+}\right)=\infty$.

Theorem 5.1. For every $\theta \in(0,1]$, the density $\rho^{(\theta)}(x)$ of the self-similar profile $F^{(\theta)}$ has the following properties:

(i) If $\theta \in(0,1)$, then as $x \rightarrow \infty$,

$$
\rho^{(\theta)}(x) \sim x^{-(1+\theta)} e^{\theta \gamma} \kappa^{\#}\left(x^{-\theta}\right) \frac{\theta(1-\theta)}{\Gamma(2-\theta)} .
$$

Here $\gamma$ is the Euler-Mascheroni constant and $\Gamma$ is the $\Gamma$-function.

(ii) If $\theta=1$, then as $x \rightarrow \infty$,

$$
\int_{0}^{x} y \rho^{(1)}(y) d y \sim e^{\gamma} \kappa^{\#}\left(x^{-1}\right) .
$$

Remarks. The asymptotics (5.8) imply the result of $[8$ for total mass in the case that $\kappa_{0}>0$. We will not pursue here the delicate question of the precise (exponential) decay rate of the density $\rho^{(1)}$ in this case, which was studied in [4] for $Q(z)=z^{2}$ and in the polynomial case in $\left[8\right.$. If $\kappa_{0}=0$, however, we see that $F^{(1)}$ has infinite mass.

Proof. We rewrite equation (5.2) for $\bar{F}^{(\theta)}$ using (3.14) and standard asymptotics for the exponential integral [14, as follows. With $w=1-\bar{F}^{(\theta)}(q)$,

$$
-\varphi\left(\bar{F}^{(\theta)}(q)\right)=\log (w \kappa(w))=-\theta \operatorname{Ei}(q)=\theta(\log q+\gamma+o(1)), \quad q \rightarrow 0 .
$$

Then $w \kappa(w) \sim q^{\theta} e^{\theta \gamma}$, whence asymptotic inversion [3, Sec. 1.5.7] yields

$$
w=1-\bar{F}^{(\theta)}(q) \sim q^{\theta} e^{\theta \gamma} \kappa^{\#}\left(q^{\theta}\right) .
$$

Now differentiating (i.e., using Lemma 3.3 of [10]) we find

$$
\int_{0}^{\infty} e^{-q x} x F^{(\theta)}(d x)=-\partial_{q} \bar{F}^{(\theta)}(q) \sim \theta q^{\theta-1} e^{\theta \gamma} \kappa^{\#}\left(q^{\theta}\right), \quad q \rightarrow 0 .
$$


Now the Tauberian Theorem 3.5 implies

$$
\int_{0}^{x} y F^{(\theta)}(d y) \sim \frac{\theta}{\Gamma(2-\theta)} x^{1-\theta} e^{\theta \gamma} \kappa^{\#}\left(x^{-\theta}\right), \quad x \rightarrow \infty,
$$

which is just (5.8) in the case $\theta=1$.

We can now also derive the decay behavior of $\rho^{(\theta)}$ in the case $\theta<1$. In fact, it follows from (5.4) that $y \rho^{(\theta)}(y)$ is decreasing. Hence by a lemma in [7, XIII.5], (5.10) implies (5.7).

\section{Domains OF ATtRACTION OF SELF-SIMILAR SOLUTIONS}

We proceed to prove Theorem 1.1 The proof has two parts. The first is to show that regular variation of $l(t)$ as $t \rightarrow \infty$ is necessary and sufficient for convergence to a scaling limit. The second is the equivalence between regular variation of $l(t)$ as $t \rightarrow \infty$ and regular variation of $\int_{0}^{x} y F_{t_{0}}(d y)$ as $x \rightarrow \infty$. The second part is based on the Tauberian Theorems 3.5 and 3.6. The most subtle aspect (despite the simple proof) is to deduce the regular variation of $l$ from the existence of a scaling limit. This is the assertion of rigidity, and we treat it first.

6.1. Regular variation of the min history is necessary. 1. Assume there is a rescaling $\lambda(t) \rightarrow \infty$ and a probability distribution function $F_{*}$ with positive min (called $\tau_{*}$ ) such that $F_{t}(\lambda(t) \cdot) \rightarrow F_{*}$. This is equivalent to convergence of the Laplace transforms,

$$
\lim _{t \rightarrow \infty} \bar{F}_{t}\left(\frac{q}{\lambda(t)}\right)=\bar{F}_{*}(q), \quad q \geq 0 .
$$

After a trivial scaling of time and cluster size, we may assume $t_{0}=1$ and $\tau_{*}=1$. Since $F_{*}$ is a probability measure with positive min, there is a unique trace measure $A_{*}$, with $A_{*}(\tau)=0$ on $(0,1)$ and $A_{*}(\tau) \rightarrow \infty$ as $\tau \rightarrow \infty$, and a min history $l_{*}$ on $[1, \infty)$, with $l_{*}(1)=1$, such that

$$
\bar{A}_{*}(q)=\int_{1}^{\infty} e^{-q l_{*}(s)} \frac{d s}{s}=\varphi\left(\bar{F}_{*}(q)\right) .
$$

Now, $s \mapsto F_{t s}(\lambda(t) x)$ is a rescaled solution, by (4.8). The rescaled min histories given by $l^{(t)}(s)=l(t s) / \lambda(t), t, s \geq 1$ have associated trace measures $A^{(t)}$ satisfying $\bar{A}^{(t)}(q) \rightarrow \bar{A}_{*}(q)$ as $t \rightarrow \infty$, for all $q>0$. We use the solution formula (2.30), (6.1) and (6.2) to obtain

$$
\lim _{t \rightarrow \infty} \int_{1}^{\infty} e^{-q l(t s) / \lambda(t)} \frac{d s}{s}=\int_{1}^{\infty} e^{-q l_{*}(s)} \frac{d s}{s}, \quad q>0,
$$

such that at every point of continuity of $l_{*}$,

$$
\lim _{t \rightarrow \infty} l(t s) / \lambda(t)=l_{*}(s) .
$$

2. Let $s_{0}$ be a point of continuity of $l_{*}$, and let $U(t)=l_{*}\left(t s_{0}\right), t \geq 1$. Let $B$ be the set of $x \geq 1$ such that

$$
\psi(x)=\lim _{t \rightarrow \infty} \frac{U(t x)}{U(t)}
$$


exists. We deduce $\psi(x)$ is a power of $x$ by following the simple argument in 7 , VIII.8.1]: By (6.4), we have $x \in B$ if $x s_{0}$ is a point of continuity of $l_{*}$. If $x_{1}$, $x_{2} \in B$, then $x_{1} x_{2} \in B$ and

$$
\psi\left(x_{1} x_{2}\right)=\psi\left(x_{1}\right) \psi\left(x_{2}\right) .
$$

Since $U$ is increasing, so is $\psi$, and since $B$ is dense in $[1, \infty)$ we can extend $\psi$ by right continuity so (6.6) holds for all $x_{1}, x_{2} \geq 1$. Then we can set $\psi(x)=1 / \psi(1 / x)$ for $x \in(0,1)$ and have (6.6) for all $x>0$. Since $\psi(x)$ is positive, locally bounded and not constant, it follows $\psi$ is a pure power law, and we can write $\psi(x)=x^{1 / \theta}$ for some $\theta>0$.

3. Since $\psi$ is continuous and increasing, it is easy to see (6.5) holds for all $x \in[1, \infty)=B$. Then we infer $\tilde{L}(t)=U(t) t^{-1 / \theta}$ is slowly varying, and $U$, hence $l$, is regularly varying at $\infty$ with index $1 / \theta$. Further, since $l_{*}\left(x s_{0}\right) / l_{*}\left(s_{0}\right)=\psi(x)=x^{1 / \theta}$ whenever $x s_{0}$ is a point of continuity of $l_{*}$, and $s_{0}$ is an arbitrary point of continuity, it follows $l_{*}(t)=t^{1 / \theta}$ for all $t \geq 1$.

6.2. Regular variation of the min history is sufficient. Let us assume that the min history $l$ is regularly varying, as in (1.12). Convergence to self-similar form is then quick: Since

$$
\frac{l(t s)}{l(t)}=s^{1 / \theta} \frac{\tilde{L}(t s)}{\tilde{L}(t)}
$$

we have $l(t s) / l(t) \rightarrow s^{1 / \theta}$ as $t \rightarrow \infty$, locally uniformly in $s$. With $\varepsilon \in(0,1 / \theta)$ fixed, there exists $t_{\varepsilon}$ such that whenever $t>t_{\varepsilon}, \tilde{L}(t r) / \tilde{L}(t) \geq 2^{-\varepsilon}$ for all $r \in[1,2]$. Then for any $s \geq 1, s=2^{n} r$ with $r \in[1,2]$ and $n$ an integer, and

$$
\frac{\tilde{L}(t s)}{\tilde{L}(t)}=\frac{\tilde{L}\left(t 2^{n} r\right)}{\tilde{L}\left(t 2^{n}\right)} \prod_{k=1}^{n} \frac{\tilde{L}\left(t 2^{k}\right)}{\tilde{L}\left(t 2^{k-1}\right)} \geq 2^{-(n+1) \varepsilon} \geq \frac{1}{2} s^{-\varepsilon} .
$$

Therefore, we may use the solution formula and the dominated convergence theorem to see that as $t \rightarrow \infty$,

$$
\varphi\left(\bar{F}_{t}\left(\frac{q}{l(t)}\right)\right)=\int_{1}^{\infty} e^{-q l(t s) / l(t)} \frac{d s}{s} \rightarrow \int_{1}^{\infty} e^{-q s^{1 / \theta}} \frac{d s}{s} .
$$

This implies $\lim _{t \rightarrow \infty} F_{t}(l(t) x)=F^{(\theta)}(x)$ for every $x>0$, where $F^{(\theta)}$ is a probability distribution with positive min $(=1)$ and Laplace transform given by (5.2).

6.3. Tauberian arguments. We now prove that if $0<\theta \leq 1$ and $\int_{0}^{x} y F_{t_{0}}(d y)$ is regularly varying with index $1-\theta$ as $x \rightarrow \infty$, then $l(t)$ is regularly varying with index $1 / \theta$ as $t \rightarrow \infty$, and conversely.

1. It is convenient first to assume (1.11) in the form

$$
\int_{0}^{x} y F_{t_{0}}(d y) \sim \frac{\theta}{\Gamma(2-\theta)} x^{1-\theta} L_{1}(x), \quad x \rightarrow \infty,
$$

where $L_{1}$ is slowly varying.

Then Theorem 3.5 implies

$$
-\partial_{q} \bar{F}_{t_{0}}(q) \sim \theta q^{\theta-1} L_{1}\left(q^{-1}\right), \quad q \rightarrow 0 .
$$

By integration (not difficult to justify as in Lemma 3.3 of [10]) we find

$$
w:=1-\bar{F}_{t_{0}}(q) \sim q^{\theta} L_{1}\left(q^{-1}\right), \quad q \rightarrow 0 .
$$


Using the solution formula (2.30) and (3.14), we write

$$
\log (w \kappa(w))=-\varphi\left(\bar{F}_{t_{0}}(q)\right)=-\int_{t}^{\infty} e^{-q l(s)} \frac{d s}{s}=-\bar{A}(q) .
$$

Hence, $\exp (-\bar{A}(q))$ is regularly varying with exponent $\theta$, and Theorem 3.6 (de Haan's exponential Tauberian theorem) implies that

$$
e^{A(1 / q)} e^{-\bar{A}(q)}=l^{\dagger}\left(q^{-1}\right) w \kappa(w) \rightarrow e^{\gamma \theta}, \quad q \rightarrow 0 .
$$

Thus, $l^{\dagger}(\tau) \sim e^{\gamma \theta} \tau^{\theta} \hat{L}_{1}\left(\tau^{\theta}\right)$ as $\tau \rightarrow \infty$, with

$$
\hat{L}_{1}(s)=\frac{1}{L_{1}\left(s^{1 / \theta}\right) \kappa\left(s^{-1} L_{1}\left(s^{1 / \theta}\right)\right)} .
$$

It is easy to show $\hat{L}_{1}$ is slowly varying, using the uniform convergence theorem for slowly varying functions 3 , Theorem 1.2.1].

Asymptotically solving $t=l^{\dagger}(\tau)$ by inverting $s \mapsto s \hat{L}_{1}(s)$ using the de Bruijn conjugate [3, Theorem 1.5.13] finally yields

$$
l(t) \sim e^{-\gamma} t^{1 / \theta} \hat{L}_{1}^{\#}(t)^{1 / \theta}, \quad t \rightarrow \infty,
$$

which gives (1.12). If $\kappa_{0}:=\lim _{q \rightarrow 0} \kappa(q)>0$, we also can write

$$
l(t) \sim e^{-\gamma}\left(\kappa_{0} t\right)^{1 / \theta}\left(L_{1}^{-1 / \theta}\right)^{\#}(t), \quad t \rightarrow \infty .
$$

2. We now prove the converse. Assuming that (1.12) holds, then

$$
l(t) \sim e^{-\gamma} t^{1 / \theta} L_{2}(t)^{1 / \theta}, \quad t \rightarrow \infty,
$$

for some slowly varying function $L_{2}$. Then, by inversion, $l^{\dagger}(\tau) \sim e^{\gamma \theta} \tau^{\theta} L_{2}^{\#}\left(\tau^{\theta}\right)$ as $\tau \rightarrow \infty$. Since $l^{\dagger}(\tau)=\exp A(\tau)$ and $\bar{A}(q)=-\log (w \kappa(w))$ with $w=1-\bar{F}_{t_{0}}(q)$, we infer from Theorem 3.6 that (6.8) is true. This implies

$$
w \kappa(w) \sim q^{\theta} L_{2}^{\#}\left(q^{-\theta}\right)^{-1}, \quad q \rightarrow 0,
$$

and asymptotic inversion yields $w \sim q^{\theta} \hat{L}_{2}\left(q^{-1}\right)$ as $q \rightarrow 0$, with

$$
\hat{L}_{2}(x)=L_{2}^{\#}\left(x^{\theta}\right)^{-1} \kappa^{\#}\left(x^{-\theta} L_{2}^{\#}\left(x^{\theta}\right)^{-1}\right),
$$

and $\hat{L}_{2}$ is slowly varying. Differentiating using Lemma 3.3 of [10], we find

$$
\partial_{q} w=-\partial_{q} \bar{F}_{t_{0}}(q) \sim \theta q^{\theta-1} \hat{L}_{2}\left(q^{-1}\right), \quad q \rightarrow 0 .
$$

Since $-\partial_{q} \bar{F}_{t_{0}}(q)=\int_{0}^{\infty} e^{-q x} x F_{t_{0}}(d x)$ the Tauberian Theorem 3.5 implies that

$$
\int_{0}^{x} y F_{t_{0}}(d y) \sim \frac{\theta}{\Gamma(2-\theta)} x^{1-\theta} \hat{L}_{2}(x), \quad x \rightarrow \infty,
$$

which is just (1.11). If $\kappa_{0}:=\lim _{q \rightarrow 0} \kappa(q)>0$, then

$$
\int_{0}^{x} y F_{t_{0}}(d y) \sim \frac{\theta}{\Gamma(2-\theta)} \frac{x^{1-\theta}}{L_{2}^{\#}\left(x^{\theta}\right) \kappa_{0}}, \quad x \rightarrow \infty .
$$

This completes the proof. 


\section{ETERNAL SOLUTIONS}

Definition 7.1. A weak solution $F$ for min-driven clustering is an eternal solution if it is defined on the maximal interval of existence $(0, \infty)$.

Our understanding of eternal solutions is closely connected to the question of how they emerge from clusters of infinitesimal size. From the solution formula (2.30) we see that as $t$ approaches zero,

$$
\varphi\left(\bar{F}_{t}(q)\right)=\int_{t}^{\infty} e^{-q l(s)} \frac{d s}{s} \rightarrow \infty
$$

hence $\bar{F}_{t}(q) \rightarrow 1$, and this means the relative size distribution $F_{t}$ always converges to a Dirac delta at zero size. A different scaling is needed to distinguish solutions through limits as $t \downarrow 0$.

7.1. The class of g-measures. What we will show is that the class of eternal solutions is in one-to-one correspondence with a suitable space of measures that can be loosely thought of as 'rescaled initial data' at $t=0$. This correspondence parallels the classical probabilistic characterization of infinitely divisible laws via a Lévy-Khintchine formula. In probability theory, infinitely divisible distributions are parametrized by the Lévy-Khintchine representation theorem, which expresses the log of the characteristic function (Fourier transform) in terms of a measure that satisfies certain finiteness conditions. In particular [7, XIII.7], a function $\omega(q)$ is the Laplace transform $\int_{0}^{\infty} e^{-q x} F(d x)$ of an infinitely divisible probability measure $F$ supported on $[0, \infty)$ if and only if $\omega(q)=\exp (-\eta(q))$, where the Laplace exponent $\eta$ admits the representation

$$
\eta(q)=\int_{[0, \infty)} \frac{1-e^{-q x}}{x} G(d x)
$$

for some measure $G$ on $[0, \infty)$ that satisfies

$$
\int_{[0, \infty)}\left(1 \wedge y^{-1}\right) G(d y)<\infty
$$

(Here $a \wedge b=\min (a, b)$.) As in [11] we call such measures g-measures (short for "generating measures," a term motivated by their connection with generators of convolution semigroups in probability [7, XIII.9(a)]). Some basic analytic facts about Laplace exponents and g-measures are collected in [11, Sec. 3].

Definition 7.2. A measure $G$ on $[0, \infty)$ is a g-measure if (17.2) holds. In addition, we say that a g-measure $G$ is divergent if

$$
G(0)>0 \quad \text { or } \quad \int_{[0, \infty)} y^{-1} G(d y)=\infty .
$$

Here recall that we use the notation $G(x)=\int_{[0, x]} G(d y)$. The space of g-measures has a natural weak topology which is fundamental in our study of scaling dynamics.

Definition 7.3. A sequence of g-measures $G^{(n)}$ converges to a g-measure $G$ as $n \rightarrow \infty$ if at every point $x \in(0, \infty)$ of continuity of $G$ we have

$$
G^{(n)}(x) \rightarrow G(x) \quad \text { and } \quad \int_{[x, \infty)} y^{-1} G^{(n)}(d y) \rightarrow \int_{[x, \infty)} y^{-1} G(d y) .
$$


7.2. A Lévy-Khintchine formula. Our analysis of eternal solutions is motivated by a classification theorem of Bertoin for Smoluchowski's coagulation equation with additive kernel [1]. Here eternal solutions were shown to be in correspondence with divergent g-measures. This theorem was generalized to other solvable kernels in [11, based on the observation that there is a natural Laplace exponent $\eta_{t}$ associated to every solution. For the model now under study, it is determined by the g-measure

$$
G_{t}(d x)=\frac{x F_{t}(d x)}{t \kappa^{\#}(t)}
$$

and the associated Laplace exponent is

$$
\eta_{t}(q)=\frac{1-\bar{F}_{t}(q)}{t \kappa^{\#}(t)}=\int_{0}^{\infty} \frac{1-e^{-q x}}{x} G_{t}(d x) .
$$

(Recall that $\kappa^{\#}$ from (5.6) is the de Bruijn conjugate of $\kappa$ from (3.14), and that $\kappa^{\#}(0+)=\infty$ if $\sum p_{k} k \log k=\infty, \kappa^{\#}\left(0^{+}\right)<\infty$ if $\sum p_{k} k \log k<\infty$.)

Theorem 7.4. (a) Let $F$ be an eternal solution of (4.5). Then there is a divergent g-measure $H$ such that $G_{t}$ converges to $H$ as $t \downarrow 0$.

(b) Conversely, for every divergent $g$-measure $H$, there is a unique eternal solution $F$ of (4.5) such that $G_{t}$ converges to $H$ as $t \downarrow 0$.

(c) The Laplace exponent of $H$ is related to the min history $l(t)$ of $F$ by

$$
\log \eta_{*}(q)=\int_{0}^{1}\left(1-e^{-q l(s)}\right) \frac{d s}{s}-\int_{1}^{\infty} e^{-q l(s)} \frac{d s}{s} .
$$

To fix ideas, it may help to note that it will follow from (7.7) that the selfsimilar solutions $F_{t}(x)=F^{(\theta)}\left(x / t^{1 / \theta}\right)$ of section 5 , normalized to have min history $l(t)=t^{1 / \theta}$, are generated by the power-law Laplace exponents

$$
\eta_{*}(q)=e^{\theta \gamma} q^{\theta}, \quad \theta \in(0,1],
$$

where $\gamma$ is the Euler-Mascheroni constant, which satisfies [14]

$$
\gamma=\int_{0}^{1} \frac{1-e^{-s}}{s} d s-\int_{1}^{\infty} \frac{e^{-s}}{s} d s
$$

The corresponding divergent g-measures are given by

$$
H_{\theta}(x)=\frac{\theta e^{\theta \gamma}}{\Gamma(2-\theta)} x^{1-\theta}, \quad \theta \in(0,1] .
$$

Proof of Theorem 7.4. In all that follows, $q>0$ is fixed, and we use the equivalence between convergence of g-measures and pointwise convergence of Laplace exponents, as established in [11, Sec. 3], for example.

1. First, assume that $F$ is an eternal solution. We claim that for all $q>0$, $\eta_{t}(q) \rightarrow \eta_{*}(q)$ as $t \downarrow 0$, where $\eta_{*}$ is given by (7.7) and satisfies $\eta_{*}(\infty)=\infty$. (This claim is proven in Step 3.) By [11, Sec. 3] it follows that $\eta_{*}$ is the Laplace exponent of a divergent g-measure $H$ and $G_{t} \rightarrow H$ as $t \downarrow 0$.

2. We first recall from Theorem 4.4 that the min history satisfies (4.4) whenever $0<t_{0}<t$. Regarding $t$ as fixed and $t_{0}$ variable, we conclude that

$$
0<l(s)<C s^{(\log 2) / Q_{1}}, \quad 0<s<1 .
$$

It follows from this, the estimate $1-e^{-q l(s)} \leq q l(s)$, and (4.4) that the integrals in (7.7) converge, so that $\eta_{*}(q)$ is finite for $0<q<\infty$. Moreover, $\eta_{*}(0)=0$ and $\eta_{*}(\infty)=\int_{0}^{1} d s / s=\infty$. 
3. We let $w=w_{t}(q)=1-\bar{F}_{t}(q)=t \kappa^{\#}(t) \eta_{t}(q)$ and use the solution formula (2.30) together with (3.14) to write

$$
\log (w \kappa(w))=-\varphi\left(\bar{F}_{t}(q)\right)=-\int_{t}^{\infty} e^{-q l(s)} \frac{d s}{s} .
$$

Adding $-\log t=\int_{t}^{1} d s / s$ to both sides we find

$$
\log (w \kappa(w) / t)=\int_{t}^{1}\left(1-e^{-q l(s)}\right) \frac{d s}{s}-\int_{1}^{\infty} e^{-q l(s)} \frac{d s}{s}=\log \eta_{*}(q)+o(1)
$$

as $t \downarrow 0$. Hence $w \kappa(w) \sim t \eta_{*}(q)$, and asymptotic inversion yields

$$
w \sim t \eta_{*}(q) \kappa^{\#}\left(t \eta_{*}(q)\right) \sim t \eta_{*}(q) \kappa^{\#}(t)
$$

since $\kappa^{\#}$ is slowly varying. But immediately this yields $\eta_{t}(q) \rightarrow \eta_{*}(q)$ as $t \downarrow 0$, and this finishes the proof of (a) and (c).

4. We now establish the converse. Let $H$ be a divergent g-measure with Laplace exponent $\eta_{*}$ (not known at first to satisfy (17.7)). We will first establish that the Lévy-Khintchine formula (7.7) defines an appropriate min history $l$, then verify that $l$ defines an eternal solution.

First, we remark that the definition of the trace admits a natural modification for eternal solutions. Given the min history of an eternal solution, we define the trace through (3.3). Conversely, we say $A$ is a maximal trace if $A$ is a distribution function on $(0, \infty)$ such that (i) $\lim _{\tau \rightarrow 0} A(\tau)=-\infty$, (ii) $\lim _{\tau \rightarrow \infty} A(\tau)=\infty$, and (iii) $\int_{0}^{\tau_{0}} \tau A(d \tau)<\infty$ for some $\tau_{0}>0$. In this case, the min history is given by (3.4) with $t_{0}=0$.

Since $\eta_{*}$ is the Laplace exponent of a g-measure, $\eta_{*}^{\prime}$ and $1 / \eta_{*}$ are completely monotone functions. Thus, there is a positive measure $A$ such that

$$
\left(\log \eta_{*}\right)^{\prime}=\frac{\eta_{*}^{\prime}}{\eta_{*}}=\int_{0}^{\infty} e^{-q \tau} \tau A(d \tau) .
$$

For every $\tau_{0}>0$, the measure $A$ satisfies the finiteness conditions

$$
\left(\log \eta_{*}\right)^{\prime} \geq\left\{\begin{array}{l}
e^{-q \tau_{0}} \int_{0}^{\tau_{0}} \tau A(d \tau) \\
\tau_{0} \int_{\tau_{0}}^{\infty} e^{-q \tau} A(d \tau)
\end{array}\right.
$$

Therefore, we may integrate (7.15) between $q$ and $q_{1} \in(0, \infty)$ and rearrange terms to obtain

$$
\begin{aligned}
& \log \eta_{*}(q)-\int_{0}^{\tau_{0}}\left(1-e^{-q \tau}\right) A(d \tau)+\int_{\tau_{0}}^{\infty} e^{-q \tau} A(d \tau) \\
& \quad=\log \eta_{*}\left(q_{1}\right)-\int_{0}^{\tau_{0}}\left(1-e^{-q_{1} \tau}\right) A(d \tau)+\int_{\tau_{0}}^{\infty} e^{-q_{1} \tau} A(d \tau):=C\left(q_{1}, \tau_{0}\right) .
\end{aligned}
$$

The right-hand side is independent of $q$. We let $q \rightarrow \infty$ on the left-hand side, and use $\eta(\infty)=\infty$ to see that $\int_{0}^{\tau_{0}} A(d \tau)=\infty$. Similarly, we let $q \rightarrow 0$ and use $\eta(0)=0$ to see that $\int_{\tau_{0}}^{\infty} A(d \tau)=\infty$. Thus, $A$ defines a maximal trace. Let $l$ denote the associated min history given by $l=\exp \left(A^{\dagger}\right)$.

5. Since $\tau_{0}>0$ is arbitrary, we may suppose $\tau_{0}$ is in the range of $l$ and $l\left(t_{0}\right)=\tau_{0}$. We change variables in (7.17) to obtain

$$
\log \eta_{*}(q)-\int_{0}^{1}\left(1-e^{-q l(s)}\right) \frac{d s}{s}+\int_{1}^{\infty} e^{-q l(s)} \frac{d s}{s}=C+\log t_{0} .
$$


In order to obtain the Lévy-Khintchine formula in the form (7.7), we just replace $l$ by the rescaling $\hat{l}(s)=l(a s)$, where $\log a=C+\log t_{0}$.

6. It remains to check that the solution formula (2.30) defines a solution for every $t>0$. This is proven by an approximation argument. We consider a sequence of finite g-measures $G_{n}$ that converge to the divergent g-measure $H$. We may suppose that $c_{n}:=\int_{0}^{\infty} x^{-1} G_{n}(d x) \geq n$. Let $t_{n}=1 / c_{n}$ so that $0<t_{n} \leq n^{-1}$. We will show that the sequence of solutions $F^{(n)}$ defined for $t \geq t_{n}$, with initial data given by the probability measures

$$
F_{n}(d x):=t_{n} \kappa^{\#}\left(t_{n}\right) x^{-1} G_{n}(d x),
$$

converges to a solution $F$ satisfying (2.30).

7. Let $\eta_{n}$ be the Laplace exponent of $G_{n}$; then $\eta_{n}(q) \rightarrow \eta_{*}(q)$ for $q>0$. Define

$$
w_{n}(q):=1-\bar{F}_{n}(q)=t_{n} \kappa^{\#}\left(t_{n}\right) \eta_{n}(q) .
$$

This is related to the min history $l_{n}$ determined from $F_{n}$ by the solution formula as in (7.12), namely,

$$
\log \left(w_{n} \kappa\left(w_{n}\right) / t_{n}\right)=\int_{t_{n}}^{1}\left(1-e^{-q l_{n}(s)}\right) \frac{d s}{s}-\int_{1}^{\infty} e^{-q l_{n}(s)} \frac{d s}{s} .
$$

Since $w_{n}(q) \sim t_{n} \eta_{n}(q) \kappa^{\#}\left(t_{n} \eta_{n}(q)\right)$ as $n \rightarrow \infty$, asymptotic inversion yields $w_{n} \kappa\left(w_{n}\right)$ $\sim t_{n} \eta_{n}(q)$, and then (7.20) yields

$$
\log \eta_{*}(q)=\lim _{n \rightarrow \infty} \int_{t_{n}}^{1}\left(1-e^{-q l^{(n)}(s)}\right) \frac{d s}{s}-\int_{1}^{\infty} e^{-q l^{(n)}(s)} \frac{d s}{s}
$$

Convergence of completely monotone functions also implies convergence of all derivatives. Thus,

$$
\frac{\eta_{*}^{\prime}}{\eta_{*}}=\lim _{n \rightarrow \infty} \int_{t_{n}}^{\infty} e^{-q l^{(n)}(s)} \frac{l^{(n)}(s)}{s} d s .
$$

It follows that the inverse functions $l^{(n)} \rightarrow l$ at all points of continuity. Therefore, we may let $n \rightarrow \infty$ in the solution formula

$$
\bar{F}_{t}^{(n)}(q)=\varphi^{-1}\left(\int_{t}^{\infty} e^{-q l^{(n)}(s)} \frac{d s}{s}\right)
$$

to see that $F$ defines an eternal solution.

\section{REFERENCES}

1. Jean Bertoin, Eternal solutions to Smoluchowski's coagulation equation with additive kernel and their probabilistic interpretations, Ann. Appl. Probab. 12 (2002), no. 2, 547-564. MR 1910639

2. Jean Bertoin and Jean-Francois Le Gall, Stochastic flows associated to coalescent processes. III. Limit theorems, Illinois J. Math. 50 (2006), no. 1-4, 147-181 (electronic). MR.2247827 (2008c:60032)

3. N. H. Bingham, C. M. Goldie, and J. L. Teugels, Regular variation, Encyclopedia of Mathematics and its Applications, vol. 27, Cambridge University Press, Cambridge, 1987. MR 88i:26004

4. Jack Carr and Robert L. Pego, Self-similarity in a coarsening model in one dimension, Proc. Roy. Soc. London Ser. A 436 (1992), no. 1898, 569-583. MR1177577 (93f:82032)

5. Laurens de Haan, An Abel-Tauber theorem for Laplace transforms, J. London Math. Soc. (2) 13 (1976), no. 3, 537-542. MR0407542 (53:11315)

6. B. Derrida, C. Godrèche, and I. Yekutieli, Scale-invariant regimes in one-dimensional models of growing and coalescing droplets, Phys. Rev. A 44 (1991), no. 10, 6241-6251. 
7. William Feller, An introduction to probability theory and its applications. Vol. II., Second edition, John Wiley \& Sons Inc., New York, 1971. MR42:5292

8. T. Gallay and A. Mielke, Convergence results for a coarsening model using global linearization, J. Nonlinear Sci. 13 (2003), no. 3, 311-346. MR1982018 (2004m:35145)

9. I. Ispolatov, P. L. Krapivsky, and S. Redner, War: The dynamics of vicious civilizations, Phys. Rev. E 54 (1996), no. 2, 1274-1289.

10. Govind Menon and Robert L. Pego, Approach to self-similarity in Smoluchowski's coagulation equations, Comm. Pure Appl. Math. 57 (2004), no. 9, 1197-1232. MR2059679(2005i:82051)

11. _ The scaling attractor and ultimate dynamics for Smoluchowski's coagulation equations, J. Nonlinear Sci. 18 (2008), no. 2, 143-190. MR2386718 (2009b:37141)

12. Tatsuzo Nagai and Kyozi Kawasaki, Statistical dynamics of interacting kinks II, Physica A 134 (1986), 482-521.

13. Barbara Niethammer and Robert L. Pego, Non-self-similar behavior in the LSW theory of Ostwald ripening, J. Statist. Phys. 95 (1999), no. 5-6, 867-902. MR2000h:82065

14. F. W. J. Olver, Asymptotics and special functions, Academic Press, New York, 1974. MR 0435697 (55:8655)

Division of Applied Mathematics, Box F, Brown University, Providence, Rhode Island 02912

E-mail address: menon@dam.brown.edu

Mathematical Institute, University of Oxford, Oxford, OX1 3LB, United Kingdom

E-mail address: niethammer@maths.ox.ac.uk

Department of Mathematical Sciences and Center for Nonlinear Analysis, Carnegie Mellon University, Pittsburgh, Pennsylvania 15213

E-mail address: rpego@cmu.edu 